\title{
Centrosome amplification mediates small extracellular vesicles secretion via
}

Sophie D. Adams ${ }^{1}$, Judit Csere ${ }^{1}$, Gisela D'angelo ${ }^{2}$, Edward P. Carter ${ }^{3}$, Teresa Arnandis ${ }^{1,4}$, Martin

$$
\text { Godinho } 0^{1,5, *}
$$

${ }^{2}$ Structure and Membrane Compartments, Institute Curie, Paris Sciences \& Lettres Research University, Centre for National de la Recherche Scientifique, UMR144, Paris, France

${ }^{3}$ Centre for Tumour Biology, Barts Cancer Institute, Queen Mary University of London, Charterhouse Square, London EC1M 6BQ, UK

${ }^{4}$ Current address: Department of Pathology, School of Medicine and Dentistry, Catholic University of Valencia, 46001, Valencia, Spain.

\footnotetext{
${ }^{5}$ Lead Contact

${ }^{*}$ Correspondence should be addressed to S.A.G.: s.godinho@qmul.ac.uk
} 


\section{Summary}

Bidirectional communication between cells and their surrounding environment is critical in both normal and pathological settings. Extracellular vesicles (EVs), which facilitate the horizontal transfer of molecules between cells, are recognized as an important constituent of cell-cell communication. In cancer, alterations in EV secretion contribute to the growth and metastasis of tumor cells. However, the mechanisms underlying these changes remain largely unknown. Here, we show that centrosome amplification is associated with and sufficient to promote small extracellular vesicle $\left({ }_{s} \mathrm{EV}\right)$ secretion in pancreatic cancer cells. This is a direct result due of lysosomal dysfunction, caused by increased reactive oxygen species (ROS) downstream of extra centrosomes. Defects in lysosome function promotes multivesicular body fusion with the plasma membrane, thereby enhancing ${ }_{s} E V$ secretion. Furthermore, we find that ${ }_{\mathrm{s}} \mathrm{EV} s$ secreted in response to amplified centrosomes are functionally distinct and activate pancreatic stellate cells (PSCs). These activated PSCs promote the invasion of pancreatic cancer cells in heterotypic 3-D cultures. We propose that ${ }_{s} E V s$ secreted by cancer cells with amplified centrosomes influence the bidirectional communication between the tumor cells and the surrounding stroma to promote malignancy.

\section{Keywords}

Centrosome amplification, exosomes, extracellular vesicles, ROS, lysosomes, PDAC, stellate cells, invasion 


\section{Introduction}

A variety of human cancer types often exhibit defects in the structure and number of centrosomes,

shown that centrosome abnormalities, in particular centrosome amplification, are not mere byproducts of tumorigenesis but rather play direct roles in promoting and accelerating tumor progression [3-6]. While the full extent by which centrosome abnormalities promote tumorigenesis is still unclear, centrosome amplification can directly promote aneuploidy and cell invasion, which play important roles in malignant progression [7-9]. Recently, we reported that centrosome amplification induces the secretion of several proteins with pro-invasive properties, e.g. interleukin-8, which induces invasive behavior in neighboring cells [10]. This altered secretion is partially due to a stress response that results from increased ROS downstream of centrosome amplification [10]. Thus, the presence of amplified centrosomes can also influence tumors in a non-cell autonomous manner, via protein secretion, suggesting a broader and more complex role for these abnormalities in cancer.

Secretion of cytokines, growth factors and extracellular vesicles (EVs) promote bidirectional communication between cancer cells and the tumor microenvironment (TME). This cross-talk impacts tumor initiation, progression and patient prognosis [11, 12]. EVs are membrane-bound vesicles containing proteins, lipids, DNA and RNA species (microRNA, mRNA and long non-coding RNAs) that communication is of particularly interesting due to their long-lasting effects and ability to influence distant tissues, e.g. during pre-metastatic niche formation [14]. Eukaryotic cells secrete two main types of EVs, microvesicles and exosomes, which differ in their size and biogenesis pathways. Microvesicles (large EVs, LEVs, 100-1000 nm diameter) are formed through outward budding or "shedding" of the plasma membrane. In comparison, exosomes (small EVs, sEVs, 30-150 nm diameter) are generated intracellularly as intraluminal vesicles (ILVs) within multivesicular bodies (MVBs), which are released upon the fusion of the MVBs with the plasma membrane [13]. Both types 
of EVs are secreted by cancer cells and have been shown to play key roles in tumor progression, potentially via changes in their composition $[15,16]$.

Exosomes, a subtype of ${ }_{\mathrm{S}} \mathrm{EVs}$, are critical in shaping the TME [16]. This is particularly clear in the stromal compartment, where cancer-derived exosomes can activate fibroblasts through transfer of molecules such as TGF- $\beta$ [16-19]. Fibroblast activation leads to the deposition of extracellular matrix (ECM), tumor fibrosis and metastasis [20]. This is particularly important in pancreatic cancer, where activation of the myofibroblast-like stellate cells, and consequent fibrosis, are the major contributors to the highly aggressive nature of these tumors and poor treatment efficacy [21-23]. While some exosomal components are known to contribute to fibroblast activation and recruitment (e.g. TGF- $\beta$ and Lin28B) $[19,24]$, the pathways responsible for alterations in their packaging and secretion in cancer cells remain largely unknown.

92

Here, we show that the presence of extra centrosomes is sufficient to increase secretion of ${ }_{s} E V s$, but not large ${ }_{L} E V s$. Characterization of these ${ }_{s} E V s$ by immunoelectron microscopy (IEM) and SILAC proteomic analyses suggests that they are of endocytic origin and thus enriched for exosomes. Mechanistically, we found that disruption of lysosome function, as a consequence of increased ROS in cells with extra centrosomes, prevents efficient lysosome and MVB fusion, leading to sEV secretion. Furthermore, $\mathrm{sEV}_{\mathrm{s}}$ secreted by cancer cells with extra centrosomes are functionally distinct and can induce PSC activation. Consequently, pancreatic stellate cells (PSCs) pre-treated with ${ }_{s} E V s$ from cancer cells with extra centrosomes promote invasion of pancreatic ductal adenocarcinoma (PDAC) cells in heterotypic 3-D cultures. Our findings demonstrate that centrosome amplification promotes quantitative and qualitative changes in secreted ${ }_{s} \mathrm{EV} s$ that could influence communication between the tumor and the associated stroma to promote malignancy. 


\section{Results}

\section{Centrosome amplification induces secretion of sEVs}

Our previous work demonstrated that centrosome amplification leads to proteomic changes in the secretome, including an increase in proteins associated with EVs, suggesting higher EV secretion in cells with amplified centrosomes [10]. To explore this further, we used an established ultracentrifugation (UC) method [14] to crudely separate EVs according to their size: ${ }_{L} E V s$ and $s E V s$, which we validated by nanoparticle tracking analyses (Figures S1A and S1B). To accurately measure secreted EV numbers, we used ImageStream flow cytometry to quantify fluorescently labelled EVs with the lipid dye BODIPY maleimide [25] and ensured that all serum was depleted for existing EVs by UC (Figures S1C and S1D). We found that in the mammary epithelial cell line MCF10A where the secretome analysis was previously performed [10], induction of centrosome amplification, by transient overexpression of the Polo-like kinase 4 (PLK4) in response to doxycycline (DOX) [26], led to increased secretion of ${ }_{S} \mathrm{EVS}$, but not ${ }_{\mathrm{L}} \mathrm{EV}$ s (Figure S1E).

120

Due to the well-established role of ${ }_{s} E V s$ in activating fibroblasts, and its downstream effects on pancreatic cancer prognosis and treatment $[16,22]$, we decided to investigate if the presence of extra centrosomes would impact ${ }_{s} E V$ s secretion in pancreatic cancer. To do this, we quantified the number of EVs and percentage of centrosome amplification in a panel of PDAC cell lines. We observed that cell lines with higher levels of centrosome amplification secreted increased numbers of EVs, in particular ${ }_{s} E V s$, demonstrating a significant correlation between extra centrosomes and ${ }_{s} E V$ secretion (Figures $1 \mathrm{~A}-1 \mathrm{C}$ and S1F). Furthermore, we confirmed that induction of centrosome amplification in two pancreatic cell lines, PaTu-S and HPAF-II, is sufficient to increase secretion of ${ }_{s} E V s$, but not ${ }_{L} E V s$ (Figures 1D and S1G). Additionally, depletion of SAS-6, a protein important for centrosome duplication, in cells exposed to DOX and PLK4 overexpression prevented both centrosome amplification and increased ${ }_{s} E V$ secretion, suggesting that $\mathrm{s} E \mathrm{~V}$ secretion is indeed a consequence of centrosomal alterations (Figures 1D and S1G). 
The ${ }_{s} E V s$ fractions isolated by UC were enriched for several markers associated with exosomes, such as ALIX, CD63, TSG101 and CD81 [27], but not for general membrane markers, such as flotillin (Figure 1E). We further confirmed the presence of bona fide EVs in the ${ }_{s} E V$ s fractions by EM and immunogold labeling using the SEV marker CD63 [28]. Consistent with increased ${ }_{s} E V$ secretion, we found that the percentage of $\mathrm{CD}^{\text {+ve }}$ EVs was higher in cells with extra centrosomes (+DOX) (Figure 1F). Moreover, these ${ }_{s} E V s$ were slightly larger, assessed by EM and also nanoparticle tracking analyses, suggesting that qualitative changes might also occur in these EVs (Figures $1 \mathrm{G}$ and $\mathrm{S} 1 \mathrm{H}$ ). Altogether, our results demonstrate that centrosome amplification promotes ${ }_{s} E V$ secretion.

\section{Proteomic analyses of $s E V s$ demonstrates their endocytic origin}

144 To further understand the origin and composition of these ${ }_{s} \mathrm{EVs}$, we performed stable isotope labelling by amino acids in cell culture (SILAC) proteomic analyses [29]. SILAC labelling with medium and heavy isotopes enables the exclusion of contaminant serum proteins, which would be unlabeled (equivalent of light labeling), and allows for simultaneous processing of purification steps to decrease sample-tosample variability (Figure 2A). Because UC isolated fractions can contain contaminants, such as protein aggregates and cellular debris, we further purified the ${ }_{S} E V S$ UC fraction using size exclusion chromatography (SEC) prior to proteomics analysis (Figure S2A). Commercially available qEV SEC columns designed to purify exosomes were used $[30,31]$ and ${ }_{5} E V s$ were quantified by ImageStream, as before. As expected for these columns, sEVs collected from PaTu-S.iPLK4 cells $(-/+$ extra centrosomes) eluted in fractions 7-10, with the majority eluting in fractions 8 and 9 (Figure S2B). SILAC reverse and forward labelling was performed to conduct proteomic analyses of fractions 7,8 and 9. Quantitative analyses of the proteomic data for each SEC fraction revealed that approximately 464 proteins were common to all fractions, and included known sEVs components such as ALIX, TSG101, CD81 and CD9 (Table S1). There were also proteins unique to each fraction suggesting that these ${ }_{s} E V s$ are heterogeneous (Figure 2B). Comparison of our ${ }_{\mathrm{s}} \mathrm{EV}$ proteomics data with the EV database Vesiclepedia [32] revealed that the majority of proteins in our datasets have been previously identified 
in other EV studies, confirming the robustness of our purification protocol. Enrichment analyses of common proteins present in both SILAC forward and reverse labeling experiments were performed to identify common pathways (Tables S2 and S3). Importantly, the most significantly enriched categories were associated with EV, $\mathrm{sEV}$ and linked to pathways unique for exosome biogenesis, such as recycling endosome and endocytic vesicles (Figure 2D). Moreover, pathways linked to cell communication, response to stress, pancreatic secretion and immune response were also enriched in our dataset (Figure 2D), indicating that these ${ }_{s} E V$ might have diverse functions.

To investigate if centrosome amplification impacts on ${ }_{s} \mathrm{EV}$ protein composition, we analyzed changes in the ratio of proteins present in heavy and medium labelled ${ }_{s} E V$. Protein abundance was initially median normalized to ensure that heavy and medium intensities in each sample were equivalent. Interestingly, for proteins that a SILAC ratio could be calculated for, the ratio values did not significantly change in any SEC fraction (Figure S2C and Table S4), suggesting protein composition is largely unchanged in ${ }_{s} E V$ secreted from cells with and without extra centrosomes. Overall, our SILAC data demonstrate that while extra centrosomes do not induce a major change in the ${ }_{s} E V s$ protein composition, the content of these ${ }_{s} E V s$ is consistent with an endocytic origin, indicating that this fraction is likely enriched for exosomes.

\section{Impaired lysosomal function in cells with extra centrosomes promotes $\mathrm{s} E V s$ secretion}

MVBs are generally destined for degradation, by fusion with the lysosomal compartment, or are

trafficked to the cell periphery where they fuse with the plasma membrane, resulting in exosome secretion [27, 33]. Lysosome dysfunction can shift the fate of MVBs targeted for degradation to fusion with plasma membrane, leading to increased sEVs secretion (Figure 3A) [34-36]. We demonstrated previously that centrosome amplification increases ROS [10], which can disrupt lysosomal function $[37,38]$. Therefore, we hypothesized that defective lysosomal degradation of MVBs could lead to increased ${ }_{s}$ EVs secretion in cells with amplified centrosomes (Figure $3 \mathrm{~A}$ ). To test this, we first assessed whether induction of centrosome amplification led to increased ROS production in PDAC cell lines. 
Indeed, induction of extra centrosomes increased ROS in both PaTu-S.IPLK4 and HPAF-II.IPLK4 cell

lines, as measured by the ratio of reduced versus oxidized glutathione, where a decrease indicates higher ROS levels. Increased ROS can be abolished by treating cells with the ROS scavenger N-acetyl cysteine (NAC), while hydrogen peroxide $\left(\mathrm{H}_{2} \mathrm{O}_{2}\right)$ is sufficient to increase ROS levels in these cells (Figures 3B and S3A). Using Magic Red fluorescence intensity to assess the function of the lysosomal cathepsin B protease [39], we found that cells with extra centrosomes have compromised lysosomal function. Treating cells with NAC prevented this defect, indicating that it was ROS dependent (Figures 3C, 3D and S3B). Furthermore, levels of LAMP1, a lysosomal marker, did not change in cells with extra centrosomes or in response to increased ROS (Figures S3C-3E), suggesting that ROS specifically impair lysosome function, consistent with their role in disrupting the integrity of lysosomal membranes [37]. Next, we analyzed ${ }_{s} E V s$ secretion in response to ROS. These analyses revealed that whilst increased ROS were sufficient to increase $s E V s$ secretion in PDAC cells, preventing higher ROS production in cells with amplified centrosomes, using NAC, abolished enhanced ${ }_{s} E V s$ secretion (Figure 3E). These results suggest that compromised lysosome function in cells with amplified centrosomes leads to ${ }_{s} E V s$ secretion. In agreement, inhibition of lysosome function with the vacuolar proton pump inhibitor Bafilomycin A1, which impairs lysosome acidification [40], was sufficient to increase sEVs secretion (Figures S3F-S3H) [41].

Next, we investigated if ROS could prevent fusion between MVBs and lysosomes, thereby promoting MVB fusion with the plasma membrane and release of ${ }_{s} E V s$ (Figure $3 \mathrm{~A}$ ). Using an antibody against phospholipid lysobisphosphatidic acid (LBPA), a lipid enriched at the membranes of late endosomes and MVBs [42], and lysotracker as a pH-based dye for functional lysosomes [43], we quantified the colocalization of MVBs and lysosomes in the different conditions. Centrosome amplification decreased the number of lysotracker-positive intracellular vesicles in a ROS-dependent manner but not LBPApositive intracellular vesicles, further supporting defective lysosomal function as consequence of 212 centrosome amplification (Figures 4A-4C). Strikingly, the percentage of co-localization between MVBs and lysosomes was significantly decreased in cells with extra centrosomes. NAC treatment restored 
lysosome function and MVB-lysosome co-localization in cells with extra centrosomes, while $\mathrm{H}_{2} \mathrm{O}_{2}$ was sufficient to decrease MVBs-lysosome co-localization (Figures 4A, 4B and 4D). Moreover, impairing lysosome function with Bafilomycin A1 dramatically reduced MVB-lysosome co-localization (Figures S4A-S4D). Taken together, our data suggest that decreased MVB-lysosome fusion as a consequence of increased ROS, and subsequent lysosome dysfunction, promotes $s$ EVs secretion in cells with supernumerary centrosomes.

sEVs secreted by cells with extra centrosomes activate pancreatic stellate cells to facilitate cancer cell invasion

Cancer-associated ${ }_{s} \mathrm{EV} s$ often carry altered cargoes, rendering them functionally distinct from ${ }_{s} E V s$

224 secreted by non-transformed cells $[15,16]$. The exact causes of these changes, however, remain elusive. In PDAC, secreted ${ }_{s} E V s$ may contribute to fibrosis through the activation of PSCs [44]. Thus, we investigated whether ${ }_{s} E V s$ secreted by PDAC cells with extra centrosomes could promote the activation of PSCs. sEVs collected from PDAC cells -/+ extra centrosomes (donor cells) were added to PSCs (Figures $5 \mathrm{~A}$ and $5 \mathrm{~B}$ ). Equal numbers of ${ }_{s} \mathrm{EV}$ s were added per condition to ensure that any differences observed were not due to the number of secreted ${ }_{s} E V$ s. Activation of PSCs cells was assessed by immunofluorescence of alpha smooth muscle actin ( $\alpha \mathrm{SMA})$. Increased expression and association of $\alpha$ SMA with stress fibers is a common feature of PSC activation towards a myofibroblast232 like phenotype [45] (Figure 5C). Interestingly, treatment of PSCs with ${ }_{s} E V s$ secreted by PDAC cells with extra centrosomes led to activation of $\sim 25-30 \%$ of the cell population (Figure 5D). It is important to note that by normalizing ${ }_{s} E V s$ numbers, we are likely underestimating the differences between ${ }_{s} E V s$ secreted by cells $-/+$ centrosome amplification. As a positive control, PSCs were treated with TGF- $\beta$, a well-established activator of PSCs, known to lead to a strong activation phenotype (Figures S5A and S5B) [46]. To validate these results, we further purified the ${ }_{s} E V s$ by SEC (Figures S2B and S5C) and tested the activation potential of the different isolated fractions. Not only were the ${ }_{s} E V s$ harboring the potential to activate PSCs retained after SEC fractionation, but these ${ }_{s} E V s$ associated mainly with 
one fraction (SEC8 for PaTu-S.iPLK4 and SEC9 for HPAF-II.iPLK4) (Figure 5E), further supporting the idea that secreted ${ }_{s} E V s$ are indeed heterogeneous.

Fibroblast activation is a common feature of cancer and can promote cancer cell invasion through various mechanisms including ECM remodeling and proteolysis [47]. To determine the functional relevance of PSC activation by ${ }_{s} E V s$ secreted by PDAC cells with amplified centrosomes, we investigated their impact on PDAC cell invasion. To do so, we used 3-D heterotypic cultures of HPAFII cells that form spheroids in 3-D with PSCs (Figure 6A) [48]. In contrast to non-treated PSCs, or PSCs pre-treated with ${ }_{s} E V s$ from cells with normal centrosome numbers, PSCs pre-treated with ${ }_{s} E V s$ harvested from cancer cells with extra centrosomes significantly induced invasion (Figures 6B and 6C). TGF- $\beta$ pre-treated PSCs, used as positive control, showed higher invasion potential, consistent with the stronger levels of PSC activation observed (Figures 6B, 6C and S5B). Confocal imaging of 3-D spheroids composed of cancer cells expressing $\mathrm{H}_{2} \mathrm{~B}-\mathrm{RFP}$ and PSCs expressing $\mathrm{H}_{2} \mathrm{~B}$-GFP revealed that activated PSCs lead the invasive front (Figure 6D). Our findings demonstrate that sEVs secreted by PDAC cells with extra centrosomes are functionally different and can induce PSCs activation to promote cancer invasion. 


\section{Discussion}

In this study, we demonstrate that centrosome amplification induces secretion of ${ }_{s} E V s$ that activate PSCs promoting the invasion of cancer spheroids. Activated PSCs are major players in the development of the pancreatic cancer stroma and associated fibrosis [21-23], suggesting a role for centrosome amplification in shaping the pancreatic cancer TME. Our data support a model whereby elevated ROS levels induced by extra centrosomes lead to loss of lysosomal function, favoring MVBs fusion with the plasma membrane and ${ }_{s} \mathrm{EV}$ s secretion (Figure 7).

Lysosomes are signaling centers that integrate many cellular responses to changes in nutrients, growth factors and stresses [49]. Fusion of lysosomes with autophagosomes is critical during autophagy, a self-degradative process important for the removal of protein aggregates, damaged organelles and intracellular pathogens [49]. Interestingly, centrosome amplification was recently shown to disrupt autophagy, rendering these cells sensitive to autophagy inhibitors [50]. Whether lysosome dysfunction is responsible for the autophagy defects observed in these cells is currently unknown. However, it is reasonable to assume that ROS-mediated lysosomal dysregulation could have a broader impact on the physiology of cells carrying centrosomal abnormalities.

${ }_{s} E V s$ secreted by cells with extra centrosomes exhibit many characteristics of exosomes: correct size range $(30-150 \mathrm{~nm})$ and proteomic profiling revealed an enrichment for proteins associated with exosomes and exosome biogenesis. Sub-fractionation of secreted ${ }_{s} E V s$ by SEC demonstrated not only the existence of different sub-types of ${ }_{s} E V s$, as previously reported $[51,52]$, but that functional differences between these different ${ }_{s} \mathrm{EV}$ populations also exist, as assessed by their ability to activate stellate cells. How changes in ${ }_{5} E V s$ composition occur and how these induce stellate cell activation, however, remains elusive. One possibility is that changes in the ${ }_{s} E V$ cargoes (proteins, RNA species) could be involved in stellate cell activation. Indeed, whilst SILAC ratio values for most detected proteins remained unchanged, we identified a number of proteins that were only identified in one label, for which a SILAC ratio could not be calculated. Therefore, it is possible that some of these 
proteins could play a role in PSC activation, but further studies will be required to assess if this is the case.

296

Alternatively, the presence or absence of specific proteins could influence ${ }_{s} E V$ uptake and indirectly contribute to PSC activation. Cargo transfer by EVs can be mediated by delivery of surface proteins to membrane receptors, fusion with the plasma membrane, micropinocytosis, phagocytosis and receptor-mediated endocytosis to deliver their content [53]. In addition, interaction between EVs and secreted proteins has been shown to modulate their uptake, highlighting the complex regulation of this process [54]. Tetraspanins, such as CD9, CD63 and CD81, have been shown to be involved in the interplay between adhesion molecules and integrins to promote ${ }_{s} \mathrm{EV}$ uptake [55]. The presence of specific tetraspanins could also influence the specificity of target cells. For example, sEVs lacking the expression of the tetraspanin CD63 were found to be preferentially endocytosed by neurons [56]. Interestingly, we found that CD81 was the only protein absent specifically in the ${ }_{s} E V s$ harvested from PDAC cells with amplified centrosomes that activate PSCs. Similarly, loss of CD81 has previously been reported in ${ }_{s} E V s$ that are secreted upon induction of lysosome dysfunction [35]. Whilst the reason for this CD81 loss in response to lysosomal dysregulation is currently unknown, the striking similarity suggests a common response to lysosomal defects that could potentially modulate ${ }_{s} E V$ uptake.

In summary, we describe a mechanism by which a stress response downstream of extra centrosomes culminates with the secretion of functionally different ${ }_{s} E V s$ by diverging the fate of MVBs. Several cellular stresses have been shown to induce EV secretion, such as oxidative stress, hypoxia and radiation-induced cell stress [57]. Thus, it is possible that in response to multiple stressors, MVBs that are normally targeted for lysosomal degradation play a role in the release ${ }_{s} E V s$ carrying protective functions in order to maintain tissue homeostasis. Indeed, oxidative stress itself has been shown to induce changes in the mRNA content of exosomes secreted by mouse mast cells, which help to protect the surrounding cells by conferring resistance to subsequent oxidative insult [58]. Understanding how 
stress communication protects cancer cells could allow us to exploit these mechanisms to prevent cancer cell adaptation.

\section{Acknowledgements}

We are grateful to Judith Simon and all the members of the Godinho lab for comments or discussion of the manuscript; Andrea Lafuente for providing the Dotplots used in Figure 2D; Hefin Rhys for helping with ImageStream and Nanosight; Pedro Monteiro for advice on image analyzes. This work was supported by a Cancer Research UK Centre Grant to Barts Cancer Institute (C355/A25137). E.P.C.

and R.G. were funded by Cancer Research UK (C10847/A27781). G.R. is supported by the French Government (ANR) through the 20 Investments for the Future LABEX SIGNALIFE (ANR-11-LABX-002801) and by the Fondation pour la Recherche Medicale (DEQ2011104211324). F.M. is supported by a Medical Research Council (MRC) Career Development Award (MR/P009417/1) and a Barts Charity grant (MGU0346). S.D.A. was supported by an MRC PhD studentship and J.C. supported by a Barry Reed PhD studentship. S.A.G. is a fellow of the Lister Institute and is supported by the MRC (MR/T000538/1).

\section{Author Contributions}

Conceptualization: S.D.A., S.A.G.; Methodology: S.D.A., T.A., G.D., E.P.C., R.G., G.R., F.M., S.A.G.; Validation: S.D.A., J.C.; Formal Analysis: S.D.A., J.C., G.D., F.M., S.A.G.; Investigation: S.D.A., J.C., T.A., G.D., E.P.C., M.D.; Resources: H.K., R.G., G.R., F.M., S.A.G.; Data Curation: S.D.A., J.C., F.M.; Writing -

Original Draft: S.A.G.; Writing - Review \& Editing: S.A.D., G.D., E.P.C., H.K., R.G., G.R, F.M., S.A.G.; Visualization: S.A.D., J.C., G.D., E.P.C., S.A.G.; Supervision: H.K., G.R., F.M., S.A.G.; Project Administration: S.A.G.; Funding Acquisition: S.A.G.

\section{Declaration of Interests}

The authors declare no competing interests. 


\section{Methods}

\section{Cell culture}

Adherent cell lines were cultured at $37^{\circ} \mathrm{C}$ and $5 \%$ humidified $\mathrm{CO}_{2}$. The pancreatic cancer cell lines PaTu8988t (PaTu-T; gift from Y. Wang, BCl-QMUL) PaTu-8988s (PaTu-S), Capan-1, PANC-1, CFPAC-1, HPAFII, MIA-PaCa-2 and DEC-hTERT (derived from normal pancreas) (gifts from $\mathrm{H}$. Kocher, BCl-QMUL) were grown in DMEM supplemented with $10 \% \mathrm{FBS}$ and $1 \%$ penicillin and streptomycin. HPDE cells (derived

from normal pancreas) (gift from $\mathrm{H}$. Kocher, BCI-QMUL) were grown in keratinocyte-SFM (1X) serum free media $+30 \mu \mathrm{g} / \mathrm{ml}(\mathrm{BPE})+0.2 \mathrm{ng} / \mathrm{ml} \mathrm{rEGF}$. The pancreatic stellate cell lines PS1 (gift from H. Kocher, BCl-QMUL) [59] were grown in DMEM/F12 supplemented with $10 \% \mathrm{FBS}$ and $1 \%$ penicillin and streptomycin. $5 \mathrm{ng} / \mathrm{ml}$ of recombinant TGF- $\beta$ (Peprotech) was used to treat PS1 cells for 72 hours.

Tetracycline-free FBS was used to grow cells expressing the PLK4 Tet-inducible construct. STR profiling was performed for cell line authentication on the following lines: PaTu-S, PaTu-T, Capan-1, MIA-PaCa2, Panc-1 and PS1.

\section{Chemicals}

Chemicals and treatments were performed as follows: $2 \mu \mathrm{g} / \mathrm{ml}$ Doxycycline hyclate (DOX; Sigma) for 48 hours, $100 \mu \mathrm{M} \mathrm{H}_{2} \mathrm{O}_{2}$ (Sigma) for 48 hours, $5 \mathrm{mM} \mathrm{N}$-acetyl cysteine (NAC; Sigma) for 48 hours and 20 nM Bafilomycin A1 (Sigma) for 24 hours.

\section{Lentiviral production and Infection}

To generate lentivirus, HEK-293 cells were plated in antibiotic free medium. Transfection of the appropriate lentiviral plasmid in combination with Gag-Pol (psPAX2, Addgene, 12260) and VSV-G (VSVG: pMD2.G, Addgene, 12259) was performed using lipofectamine $2000^{\circledR}$ (Thermo Fisher Scientific), as per the manufacturer's specifications. The resultant lentivirus was harvested 24 hours and 48 hours post infection, passed through a $0.4 \mu \mathrm{M}$ syringe filter and stored in cryovials at $-80^{\circ} \mathrm{C}$. For infection, the appropriate lentivirus was then mixed with $8 \mu \mathrm{g} / \mathrm{ml}$ polybrene before being added to the cells in 

after final infection.

Cells expressing the inducible PLK4 construct were generated as previously described [26]. Briefly, cells were initially infected with pLenti-CMV-TetR-Blast lentiviral vector (Addgene, 17492) and selected using Blasticidin $(10 \mu \mathrm{g} / \mathrm{ml})$. Post-selection, cells were then infected with a lentiviral vector containing PLK4 CDNA which had been previously cloned into the pLenti-CMV/TO-Neo-Dest vector and selected using Geneticin $(200 \mu \mathrm{g} / \mathrm{ml})[26,60]$. Cells expressing the PLK4 transgene were then induced for 48 hours using $2 \mu \mathrm{g} / \mathrm{ml}$ of Doxycycline.

To generate H2B-RFP iPLK4 cells, lentivirus was produced by transfecting HEK-293 cells with LV-RFP (Addgene 26001), pMD2.G (Addgene, 12259) and $\mu$ g pCMVDR8.2 (Addgene, 12263) using FuGENE (Promega, E2311), as per manufacturer's instructions. 24 hours later the medium was replaced and 48 hours post transfection the viral supernatant was collected, passed through a $0.4 \mu \mathrm{M}$ syringe filter and stored in cryovials at $-80^{\circ} \mathrm{C}$. Cells were transduced with the lentivirus as described above.

\section{SIRNA}

siRNA transfection was performed in antibiotic free growth medium using Lipofectamine ${ }^{\circledR}$ RNAiMAX as per the manufacturer's specifications. For SAS-6 knock down experiments siNegative control (siNegative, Qiagen, 1027310) and siSAS-6 (siSAS6 on-TARGET smart pool, Dharmacon, M-004158-02) were used. Per 6 well, 20 nM of siRNA was used for PaTu-S.iPLK4 cells and 50 nM for HPAF-II.iPLK4 cells as PaTu-S.iPLK4 cells were more sensitive to SAS-6 depletion and to prevent loss of centrioles below control conditions. 24 hours post transfection, the cells were trypsinized and seeded onto coverslips for analysis by immunofluorescence or into $15 \mathrm{~cm}$ dishes for exosome harvest experiments 72 hours post transfection. 


\section{Immunofluorescence 2D}

Cells plated on glass coverslips were treated for up to 48 hours with the appropriate drug treatments,

before being washed twice in PBS and fixed in 4\% Formaldehyde for 20 minutes at room temperature.

For centrin2 staining, cells were fixed in ice-cold methanol for 10 minutes at $-20^{\circ} \mathrm{C}$. Following fixation, cells were permeabilized in $0.2 \%$ Triton $\mathrm{X}-100$ in PBS for 5 minutes then blocked for 30 minutes in blocking buffer (PBS, 5\% BSA, 0.1\% Triton X-100). Cells were then incubated with primary antibody diluted in blocking solution for 1 hour. Cells were then washed with PBS and incubated with speciesspecific Alexa-conjugated secondary antibodies diluted in blocking buffer for 1 hour. Alexa Fluor 568 Phalloidin (1:250) was incubated in blocking solution for 1 hour. Cells were washed in PBS and DNA was stained with Hoechst 33342 diluted in PBS (1:5000) for 5 minutes. Finally, coverslips were mounted using ProLong ${ }^{\mathrm{TM}}$ Gold Antifade Mountant. Antibodies used included: Anti-centrin 2 N-17-R (Santa Cruz; 1:100), Anti $\alpha$-tubulin DM1 $\alpha$ (Sigma-Aldrich; 1:1000), Anti LBPA 6C4 (Merck Millipore; 1:100), Anti LC3B (D11) XP ${ }^{\circledR}$ (Cell Signalling; 1:200), Anti $\alpha$-SMA (Sigma-Aldrich; 1:300), Anti-Rabbit Alexa Flour 488 (Life Technologies; 1:1000), Anti-Rabbit Alexa Fluor 568 (Life Technologies 1:1000), Anti-Mouse Alexa Fluor 488 (Life Technologies 1:1000). Centrosome amplification was defined as the percentage of metaphase cells containing extra centrosomes ( $>4$ centrioles per cell).

Images were acquired using an inverted Nikon microscope coupled with a spinning disk confocal head (Andor). Unless otherwise stated, imaging of cancer cells was performed using a 100x objective and imaging of stellate cells with a 40x objective. Images/projection images (from z-stacks) were subsequently generated and analyzed with Image J (National institute of Health, Bethesda, MD, USA) [61]. Where Z-stack images were required to analyze fluorescence intensity, Z-stack parameters were determined using the following equation: $Z \min =1.4 \lambda \mathrm{n} /(\mathrm{NAobj}) 2 . \lambda=$ the emission wavelength, $\mathrm{n}=$ refractive index of the immersion media, $\mathrm{NAobj}=$ the numerical aperture of the objective. This equation calculates the ideal $z$ stack step size to minimize overlap between each step of the stack. Sum intensity projection images were subsequently generated using Image $J$ and fluorescence intensity was quantified using Image J. All conditions were quantified blindly. 


\section{Extracellular Vesicle (EV) Isolation}

Cells were grown for 48 hours in medium supplemented with EV depleted FBS. Vesicle depletion in FBS was performed via ultracentrifugation at $100,000 \times \mathrm{g}$ at $4^{\circ} \mathrm{C}$ for 18 hours. Where induction of centrosome amplification was necessary, cells were treated with DOX for 48 hours, before cells were washed in PBS and subsequently cultured in EV depleted media. Where drug treatments were required, cells were treated for the duration of the EV harvest ( 48 hours post addition of EV depleted media). After 48 hours, conditioned medium was collected, and a final cell count was performed to ensure the final cell count remained the same between cell types and conditions.

432 Serial ultracentrifugation (UC). Extracellular vesicles were isolated from the conditioned media via serial ultracentrifugation steps at $4^{\circ} \mathrm{C}$, similarly to [14]. Briefly, the cell culture medium was subjected to a low speed centrifugation of $500 \times \mathrm{g}$ for 10 minutes. The supernatant was then centrifuged at $12,000 \times \mathrm{g}$ for 20 minutes to pellet the large EVs (_EVs), after removal of the supernatant the ${ }_{\mathrm{L}} \mathrm{EV}$ s were re-suspended in $500 \mu \mathrm{l}$ of PBS. The supernatant was then subjected to a high-speed ultracentrifugation at $100,000 \times \mathrm{g}$ for 70 minutes to pellet the smaller EVs (sEVs). The pellet was then washed in PBS and a second high-speed ultracentrifugation was performed at 100,000 x g for 70 minutes (Figure S1A). The isolated $s_{\mathrm{S}} \mathrm{V}$ pellet was then re-suspended in $500 \mu \mathrm{l}$ of PBS. exclusion chromatography (SEC) was performed using the qEV original izon science SEC columns (as per the manufacturer's instructions). Briefly, the SEC columns were equilibrated to room temperature and flushed with $5 \mathrm{ml}$ of buffer (PBS filtered twice through $0.22 \mu \mathrm{M}$ filters) prior to use. $500 \mu \mathrm{l}$ of concentrated exosomes (isolated by serial ultracentrifugation) was added to the top of the column and the eluted fractions were collected immediately in $500 \mu$ volumes. The column was kept topped up with buffer throughout the experiment. Fractions 7-12 containing the eluted EVs were collected. 
bioRxiv preprint doi: https://doi.org/10.1101/2020.08.19.257162; this version posted August 20,2020 . The copyright holder for this preprint (which was not certified by peer review) is the author/funder, who has granted bioRxiv a license to display the preprint in perpetuity. It is made available under aCC-BY-NC-ND 4.0 International license.

\section{Extracellular Vesicle Quantification and Analysis}

Amins ImageStream ${ }^{\circledR}$ Mark II Imaging Flow Cytometer (ImageStream). EV samples were analyzed by ImageStream as previously described [25]. Briefly, samples were prepared in $50 \mu$ l volumes, labelled with the fluorescent lipid dye BODIPY ${ }^{\circledR}$ FL Maleimide [BODIPY ${ }^{\circledR}$ FL N-(2-Aminoethyl) Maleimide] (Thermo Fisher Scientific; 1:200) and incubated at room temperature in the dark 10 minutes prior to analysis. Samples were then loaded onto the ImageStream and vesicles were acquired at a slow flow rate with 60x magnification, a $488 \mathrm{~nm}$ excitation laser (BODIPY detection) and $765 \mathrm{~nm}$ laser (side scatter). The "remove bead" function was turned off and the flow rate allowed to stabilize before acquisitions. For acquisition, the storage gate was set to collect all events and the stopping gate set to the vesicle population (low to mid BODIPY intensity and low side scatter). The stopping gate was set to ensure that at least 20,000 objects were analyzed per acquisition. Three separate acquisitions were collected per sample. Analysis was then performed using the IDEAS software. To quantify objects/ml, a graph was generated plotting channel 02 fluorescence intensity (BODIPY) against channel 12 scatter intensity (side scatter) and a vesicle gate was re-applied to select the population at the correct BODIPY and side scatter intensities to be EVs (see Figure S1C). Where necessary the gate was adjusted using the Image library to eliminate noise and artefacts from the vesicle population. The objects $/ \mathrm{ml}$ statistic was then used to quantify the number of objects $/ \mathrm{ml}$ in the gated region. The average objects $/ \mathrm{ml}$ was calculated from three separate acquisitions from each sample. a syringe pump. As previously described, isolated EVs were resuspended (UC) or eluted (SEC) in Dulbecco's PBS filtered twice through $0.22 \mu \mathrm{M}$ filters. The NS300 chamber was flushed with $0.22 \mu \mathrm{M}$ filtered deionized water and then again with $500 \mu$ l of PBS (Dulbecco's PBS filtered twice through 0.22

$472 \mu \mathrm{M}$ filters) to remove any particle matter. Using a $1 \mathrm{ml}$ syringe $400 \mu \mathrm{l}$ of EV sample was then flushed through the chamber until vesicles were visible on the camera to allow the focus and gain settings to be optimized. The sample was then injected into the flow cell at speed 50 and 3 recordings of 60 seconds each were acquired. Between samples filtered PBS was used again to flush the chamber 

software and averages of the three technical replicates were plotted per experiment.

\section{Immunolabeling electron microscopy (IEM)}

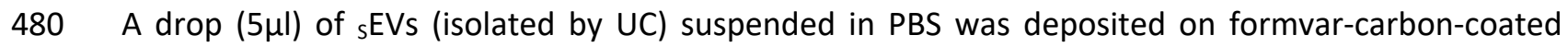
electron microscopy grids for $20 \mathrm{~min}$ at room temperature, fixed with $2 \%$ paraformaldehyde in $0.2 \mathrm{M}$ phosphate buffer ( $\mathrm{pH} 7.4)$, for $20 \mathrm{~min}$ at room temperature, and post fixed with $1 \%$ glutaraldehyde in PBS for $5 \mathrm{~min}$ at room temperature. Grids containing sEV were then washed and then blocked for 5 min at room temperature in blocking buffer (PBS, 1\% BSA). sEVs were then immunolabelled with a mouse anti-human CD63 primary antibody (Abcam ab23792) diluted in blocking solution for 1 hour at room temperature, washed with PBS, 0,1 \% BSA, incubated with a rabbit antibody against mouse Fc fragment (Dako Agilent Z0412) in PBS 0,1\% BSA for 20 min at room temperature. The preparations Department of Cell Biology, Utrecht University). Grids were analyzed on a Tecnai Spirit G2 electron microscope (Thermo Fischer Scientific) and digital acquisitions were made with a 4k CCD camera (Quemesa, Soft Imaging System). Images were analysed with iTEM software (iTEM CE Olympus serie) and data with Prism-GraphPad Prims software (v8) [62].

\section{Western Blotting}

Small extracellular vesicles harvested for protein extraction were isolated as previously described, however following the final ultracentrifugation, the pellet was lysed immediately in RIPA buffer supplemented with protease inhibitors on ice. To facilitate further lysis, samples were probe sonicated on ice. Protein concentration was determined using the Bio-Rad Protein Assay. 10 $\mu \mathrm{g}$ of protein was loaded per well. Samples were resuspended in Laemmli buffer, resolved using the NuPAGE ${ }^{\circledR}$ Bis-Tris 
bioRxiv preprint doi: https://doi.org/10.1101/2020.08.19.257162; this version posted August 20,2020 . The copyright holder for this preprint (which was not certified by peer review) is the author/funder, who has granted bioRxiv a license to display the preprint in perpetuity. It is made available under aCC-BY-NC-ND 4.0 International license.

1:1000), Anti CD81 B-11 (Santa Cruz; 1:500), Anti ALIX 3A9 (Cell Signalling; 1:1000), Anti Flotillin-1 18 (Biosciences; 1:1000), HRP- anti rabbit secondary (GE Healthcare; 1:5000) and HRP- anti mouse secondary (GE Healthcare; 1:5000). Western blots were developed on X-ray film using a SRX-101A table top film processor.

\section{Stable isotype labelling by amino acids in cell culture (SILAC)}

SILAC based proteomic analysis of exosomes was performed as previously [63]. All SILAC amino acids (heavy and medium) were purchased from Cambridge Isotopes. SILAC media and dialyzed serum were purchased from Thermo Fisher Scientific. PaTu-S.iPLK4 cells with and without the induction of centrosome amplification were grown for 6 passages in Dulbecco's modified Eagle's medium for SILAC $100,000 \times \mathrm{g}$ for EV depletion), $600 \mathrm{mg} / \mathrm{L}$ Proline and $100 \mathrm{mg} / \mathrm{L}$ of either heavy or medium Lysine and Arginine amino acids (Lys ${ }^{8}$ and $\operatorname{Arg}^{10}$ for heavy, and Lys ${ }^{4}$ and $\operatorname{Arg}^{6}$ for medium, respectively). Labelled cells were then plated at a density of $1 \times 10^{6}$ cells in 40 T175 flasks per condition. 24 hours later flasks were washed in PBS and $15 \mathrm{ml}$ of fresh EV depleted medium supplemented with the correct amino acids (heavy or medium) was added to the cells. 48 hours later, the conditioned medium was harvested and samples heavy and medium labelled were pooled together (Figure 2A). EVs were then isolated from the conditioned medium via ultracentrifugation and subsequent SEC as previously described. The experiment was then repeated with the labelling reversed.

\section{Mass spectrometry}

Extracellular vesicles were lysed in $8 \mathrm{M}$ Urea in $50 \mathrm{mM}$ Ammonium bi-carbonate ( $\mathrm{ABC})(\mathrm{pH}$ 8). Samples were then sonicated using a Diagenode Bioruptor sonicator at $4^{\circ} \mathrm{C}$. Samples were sonicated at high power for 15 cycles of 30 seconds on and 30 seconds off. $10 \mathrm{mM}$ DTT was added for 20 minutes at room temperature followed by $55 \mathrm{mM}$ lodoacetamide incubated for 30 minutes in the dark. Protein quantification was then performed as previously described. $15 \mu \mathrm{g}$ of protein was then selected per 
sample and Urea was diluted to $2 \mathrm{M}$ final concentration with $50 \mathrm{mM} \mathrm{ABC}$. Samples were then subjected to in-solution trypsin digestion overnight at $25^{\circ} \mathrm{C}$. The digested peptides were then acidified and desalted via stagetipping [64]. Peptides were then dried by vacuum centrifugation and resuspended in $10 \mu$ l of buffer $A^{*}$ ( $2 \% A C N, 0.1 \%$ trifluoroacetic acid and $0.5 \%$ acetic acid).

532

\section{LC-MS/MS analysis}

Equivalent of $\sim 1 \mu \mathrm{g}$ of each digested SILAC mix was subjected to Liquid Chromatography coupled with tandem Mass Spectrometry (LC-MS/MS), using a Q-Exactive plus Orbitrap mass spectrometer coupled with a nanoflow ultimate 3000 RSL nano HPLC platform (Thermo Fisher Scientific). Briefly, samples were resolved at a flow rate of $250 \mathrm{~nL} / \mathrm{min}$ on an Easy-Spray $50 \mathrm{~cm} \times 75 \mu \mathrm{m}$ RSLC C18 column with 2 $\mu \mathrm{m}$ particle size (Thermo Fisher Scientific), using a 123 minutes gradient of $3 \%$ to $35 \%$ of buffer-B ( $0.1 \%$ formic acid in ACN) against buffer-A ( $0.1 \%$ formic acid in water), and the separated peptides were infused into the mass spectrometer by electrospray. The spray voltage was set at $1.95 \mathrm{kV}$ and the capillary temperature was set to $255^{\circ} \mathrm{C}$. The mass spectrometer was operated in data dependent positive mode, with $1 \mathrm{MS}$ scan followed by $15 \mathrm{MS} / \mathrm{MS}$ scans (top 15 method). The scans were acquired in the mass analyzer at $375-1500 \mathrm{~m} / \mathrm{z}$ range, with a resolution of 70,000 for the $\mathrm{MS}$ and 17,500 for the

\section{Proteomics data analysis}

MaxQuant (version 1.6.3.3) software was used for database search and SILAC quantifications [65]. The search was performed against a FASTA file of the Homo Sapiens, extracted from Uniprot.org (2016). A precursor mass tolerance of $4.5 \mathrm{ppm}$, and a fragment mass tolerance of $20 \mathrm{ppm}$ was applied. Methionine oxidation and $\mathrm{N}$-terminal acetylation were included as variable modifications whilst carbamidomethylation was applied as a fixed modification. Two trypsin miss-cleavages were allowed, and the minimum peptide length was set to 7 amino acids. SILAC multiplicity was set to 3 , with Lys4 and Arg6 selected as medium, and Lys8 and Arg10 as heavy labels. Minimum SILAC ratio count was 
set at 1 . All raw files were searched together, with the match between runs option enabled. All downstream data analysis was performed by Perseus (version 1.5.5.3) [52], using the MaxQuant ProteinGroups.txt output file. Briefly, normalized SILAC H/M intensities were converted to Log 2 scale. Reverse (decoy) hits, potential contaminants, and proteins identified only by modified peptides were filtered out. Ratio values were then median subtracted. Category enrichment analysis was performed using the Fisher exact test function within Perseus. Scatter plots of the SILAC ratio values were also generated by Perseus. All mass spectrometry raw files and search results reported in this paper have been deposited at the ProteomeXchange Consortium via the PRIDE [66], with the PRIDE accession number of PXD020984.

\section{Measuring cellular reactive oxygen species (ROS)}

Cellular ROS was measured through the detection glutathione in its reduced (GSH) and oxidized (GSSG) forms using the luminescence-based GSH/GSSG-Glo ${ }^{\mathrm{TM}}$ Assay (Promega, V6611). Briefly, the Promega GSH/GSSG-Glo ${ }^{\mathrm{TM}}$ Assay is a linked assay utilizing glutathione S-transferase and Luciferin-NT GSH to GSSG can then be calculated to give a read out of oxidative stress in the cells, where a decrease in the ratio indicates an increase in oxidative stress. All reactions and calculations were carried out as per the manufacturer's instructions. The final ratio of GSH/GSSG was normalized to protein content Kit (Thermo Fisher Scientific, 23227) as per the manufacturer's instructions.

\section{Magic Red assay}

576 The Magic Red ${ }^{\text {TM }}$ Cathepsin B kit (Bio-Rad, ICT937) was used to analyze the protease activity of Cathepsin B in lysosomes as a proxy to lysosome function. In the presence of functional cathepsin B, the Magic Red substrate is cleaved allowing the Cresyl violet fluorophore to fluoresce red upon excitation at 550-590 nm. Briefly, cells to be analyzed were plated on coverslips and the Magic Red 

added to the growth media $(20 \mu \mathrm{l}$ was added per $300 \mu \mathrm{l}$ of growth media as per the manufacturer's instructions) for the final hour of the experiment. Cells were then fixed in $4 \%$ Formaldehyde as previously described. Cresyl Violet fluorescence was detected using an inverted Nikon microscope image projections were generated using Image J. Fluorescence intensity was then quantified per cell with ImageJ [61]. All conditions were quantified blindly.

\section{Extracellular vesicle uptake by recipient cells}

Fluorescently labelled EVs were generated using the previously described ultracentrifugation protocol with the following alteration: prior to the final PBS wash step, EVs were resuspended in $200 \mu \mathrm{l}$ of PBS and fluorescently labelled with BODIPY (1:200). EVs were then incubated at room temperature for 5 minutes before being diluted in $31.5 \mathrm{ml}$ of PBS. The final 100,000 $\mathrm{g}$ ultracentrifugation step was then performed, and the subsequent EV pellet resuspended in $200 \mu \mathrm{l}$ of PBS. The isolated EVs were then added to the recipient cells that had been plated on glass coverslips 24 hours prior. 3 hours post addition of EVs, coverslips were fixed in $4 \%$ formaldehyde and stained with Alexa Fluor 568 Phalloidin (1:250) and Hoechst (1:5000) as previously described. Representative z-stack images were taken using a spinning disk confocal microscope as previously described.

\section{Extracellular vesicle-mediated PSC activation assay} treatment) were cultured for 48 hours in vesicle depleted media before the conditioned media was collected. EVs were then harvested from the conditioned media by ultracentrifugation alone, or in combination with SEC as described previously. EV number was then quantified by ImageStream as 
million EVs was administered. 24 hours later cells were fixed and stained for $\alpha$-SMA and DNA as described previously. Images were acquired using an inverted Nikon microscope coupled with a spinning disk confocal head (Andor) with a 40x objective. PS1 activation was quantified based on $\alpha-$ SMA organization, where the formation of $\alpha$-SMA fibers was used as a measure of activation (maybe describe how the different categories were separated and we can reference the figure). Roughly 150 cells were quantified manually per condition. All conditions were quantified blindly.

612

\section{D co-culture spheroid invasion assay}

Prior to spheroid generation, PS1 cells were either treated for 72 hours with ${ }_{s} \mathrm{EV}$ ( (as described above), with $5 \mathrm{ng} / \mathrm{ml} \mathrm{TGF-} \beta$ or left untreated. 3D spheroid cancer cell/PS1 co-cultures were generated using a hanging drop spheroid model developed by Ed Carter and Richard Grose (BCl-QMUL), based on previous work [48]. Briefly, PS1 H2B-GFP $\left(4.4 \times 10^{4}\right.$ cells/ml) and HPAF-II.iPLK4-H2B-RFP cancer cells $\left(2.2 \times 10^{4}\right.$ cells $\left./ \mathrm{ml}\right)$ were combined in a $0.24 \%$ methylcellulose solution (Sigma-Aldrich, M0512). Droplets containing 1000 cells were then plated on the underside of a $15 \mathrm{~cm}$ culture dish and left to form spheroids overnight at $37^{\circ} \mathrm{C}$. Spheroids were then collected and centrifuged at $100 \mathrm{x} \mathrm{g}$ for 3 minutes before being re-suspended in gel mix solution. Gel mix solution consisted of $1.6 \mathrm{mg} / \mathrm{ml}$ Collagen I (Corning Rat Tail High Concentration) and $17.5 \%$ Matrigel ${ }^{\circledR}$ Matrix Basement Membrane LDEV-free (Corning, 354234), prepared in PS1 culture medium and buffered to physiological pH with $\mathrm{NaOH}$. Approximately 6 spheroids suspended in gel mix were added to a pre-coated well of a low attachment plate and left to solidify at $37^{\circ} \mathrm{C}$ before PS1 culture medium was added on top. Spheroids were incubated for 3 days and images were taken by light microscopy. Percentage invasion was analyzed using Image $\mathrm{J}$ and calculated as a measure of the total invasive area relative to the central on an LSM 880 Zeiss confocal microscope. All conditions were quantified blindly. 
bioRxiv preprint doi: https://doi.org/10.1101/2020.08.19.257162; this version posted August 20,2020 . The copyright holder for this preprint

(which was not certified by peer review) is the author/funder, who has granted bioRxiv a license to display the preprint in perpetuity. It is made available under aCC-BY-NC-ND 4.0 International license.

632

\section{Statistical Analysis}

Graphs and statistics were generated using Prism 8 (GraphPad Software) where results are presented as mean \pm standard deviation (SD) unless otherwise stated. Statistical analysis was performed using one-way ANOVA with either a Tukey's (parametric) or Kruskal-Wallis (non-parametric) post hoc test 


\section{Figure legends}

Figure 1. Centrosome amplification promotes the secretion of ${ }_{s} E V s$ in PADC cells.

(A) Representative confocal images of mitotic cells with normal and amplified centrosomes. Cells were stained for $\alpha$-tubulin (magenta), centrin2 (green) and DNA (cyan). Scale bar, $10 \mu \mathrm{m}$. (B) Quantification of ${ }_{S} E V s$ and ${ }_{L} E V s$ secreted by PDAC cell lines. Average of the percentage of centrosome amplification (CA) per cell line is highlighted in orange. (C) Linear regression of the data presented in B and Spearman correlation coefficients for ${ }_{s} E V S$ and ${ }_{L} E V S$. (D) Quantification of secreted ${ }_{s} E V s$ and ${ }_{L} E V s$ in Patu-S.iPLK4 and HPAF-II.iPLK4 cell lines upon induction of centrosome amplification (+DOX), before and after depletion of Sas- 6 by siRNA. Average percentage of centrosome amplification (CA) per condition is highlighted in orange. (E) Western blot analyses of proteins associated with ${ }_{5} E V s$ in extracts from cells and ${ }_{s}$ EVs collected by UC. (F) Top: Representative images of IEM of ${ }_{s} E V s$ collected from HPAF.iPLK4 cells. Dark beads represent immunogold labelling with anti-CD63. Scale bar, $200 \mathrm{~nm}$. Bottom: Quantification of the percentage of positive CD63 ${ }_{s} E V s$. (G) Quantification of ${ }_{s} E V s$ diameter by cry-EM. Patu-S.iPLK4 ${ }_{s} E V s n_{(-D O x)}=232$ and $n_{(+D O X)}=216 ;$ HPAF-II.iPLK4 $n_{(-D O X)}=541$ and $n_{(+D O X)}=493$. For

all graphics error bars represent mean $+/-$ SD from three independent experiments. ${ }^{*} p<0.05$, ${ }^{* *} p<0.01, * * * * p<0.0001$. The following statistic were applied: for graphs in D two-way ANOVA with Tukey's post hoc test was applied and for graphs in G unpaired $t$ test was applied. See also Figure S1.

Figure 2. Proteomic analyses of ${ }_{s} \mathrm{EVs}$ secreted by cells with extra centrosomes support their endocytic origin. (A) Experimental flowchart. (B) Venn diagram comparing the ${ }_{s} E V s$ proteomes of SEC fractions 7, 8 and 9. (C) Venn diagram comparing the depicting the ${ }_{s} E V$ s proteome of SEC fractions 7, 8 and 9 with the Vesiclepedia database. (D) Dotplot representation of the enrichment analyses performed for the common proteins in all SEC fractions. Only proteins that were identified in both forward and reverse labelling experiments were considered for this analysis. See also Figure S2 and Tables S1-S3. 
Schematic representation of intraluminal vesicle formation (ILVs) and multivesicular bodies (MVBs) fate and how ROS could affect this process. (B) Levels of intracellular ROS quantified by the ratio of GSH/GSSG in Patu-S.iPLK4 and HPAF-II.iPLK4 cell lines. Decrease in the GSH/GSSG ratio indicates higher ROS levels. $5 \mathrm{mM}$ of NAC and $100 \mu \mathrm{M} \mathrm{H} \mathrm{O}_{2}$ was used. (C) Representative confocal images of cells stained with Magic red (magenta), as a proxy for lysosome function, and for DNA (cyan). Scale bar, $10 \mu \mathrm{m}$. (D) Quantification of intracellular Magic red fluorescence intensity normalized for cell area in Patu-S.iPLK4 cells. AU, arbitrary units. $5 \mathrm{mM}$ of NAC and $100 \mu \mathrm{M} \mathrm{H}_{2} \mathrm{O}_{2}$ was used. $\mathrm{n}_{\text {(-Dox })}=158$, $n_{(+D O X)}=189, n_{(+D O X+N A C)}=221$ and $n_{(-D O X+H 2 O 2)}=175$. (E) Quantification of secreted $s_{s} E V$ and ${ }_{L} E V s$ in PatuS.iPLK4 and HPAF-II.iPLK4 cell lines. For all graphics error bars represent mean +/- SD from three independent experiments. ${ }^{*} p<0.05,{ }^{* *} p<0.01,{ }^{* *} p<0.001,{ }^{* * *} p<0.0001$, n.s. $=$ not significant $(p>0.05)$. The following statistic were applied: for graphs in B one-way ANOVA with Tukey's post hoc test, for D one-way ANOVA with a Kruskal-Wallis post hoc test and for E two-way ANOVA with Tukey's post hoc test. See also Figure S3.

Figure 4. Centrosome amplification decreases lysosome-MVBs co-localization in a ROS-dependent

manner. (A) Representative confocal images of cells stained for acidic lysosomes (Lysotracker, magenta), late endosomes/MVBs (anti-LBPA, green) and DNA (grey). Insets show higher magnification of lysotracker and LBPA-labelled vesicles. Scale bar, $10 \mu \mathrm{m}$. (B) Quantification of the number of

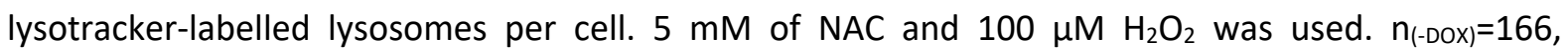

$\mathrm{n}_{(+D O X)}=182, \quad \mathrm{n}_{(+D O X+N A C)}=245 \quad$ and $\mathrm{n}_{(-\mathrm{DOX}+\mathrm{H} 2 \mathrm{O} 2)}=187$.

(C) Quantification of LBPA-labelled late endosomes/MVBs per cell. $5 \mathrm{mM}$ of $\mathrm{NAC}$ and $100 \mu \mathrm{M} \mathrm{H} \mathrm{H}_{2} \mathrm{O}_{2}$ was used. $\mathrm{n}_{(-\mathrm{DOX})}=88, \mathrm{n}_{(+\mathrm{DOX})}=102$, $n_{(+D O X+N A C)}=129$ and $n_{(-D O X+H 202)}=x 99$. (D) Quantification of the percentage of lysotracker and LBPA-

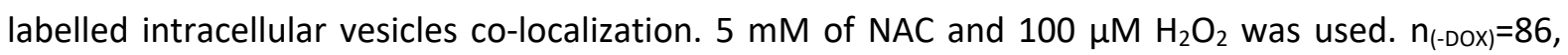


(A) Experimental flowchart. (B) Representative confocal image of PSCs incubated with ${ }_{s} E V$ s. Cells were stained for f-actin (phalloidin, grey) and DNA (cyan). Isolated sEVs were labelled with Bodipy (green). Scale bar $=10 \mu \mathrm{m}$. (C) Representative confocal images of PSCs stained for $\alpha$-SMA (green) and DNA (cyan). Scale bar, $10 \mu \mathrm{m}$. (D) Quantification of the percentage of PSCs activation upon treatment with ${ }_{s}$ EVs collected by UC from Patu-S.iPLK4 (left) and HPAF-II.iPLK4 (right), with (+DOX) and without (-DOX) extra centrosomes. Patu-S.iPLK4 isolated $s$ EV: PSCs $n_{(- \text {Dox SEVs })}=398, n_{(+D O X S E V s)}=373$, and $n_{(c t r)}=475$. HPAFII.iPLK4 isolated ${ }_{s E V}$ : PSCs $n_{(-D O X ~ S E V s)}=914, n_{(+D O x ~ S E V s)}=1057$, and $n_{(c t r)}=718$. (E) Quantification of the percentage of PSCs activation upon treatment with ${ }_{s}$ EVs collected by SEC from Patu-S.iPLK4 (left) and HPAF-II.iPLK4 (right), with (+DOX) and without (-DOX) extra centrosomes. Patu-S.iPLK4 isolated ${ }_{s} E V$ :

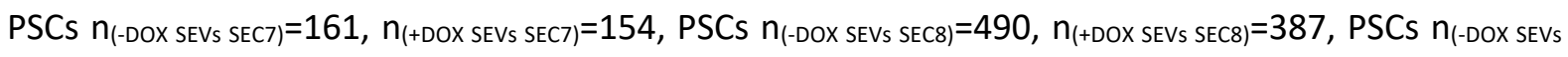

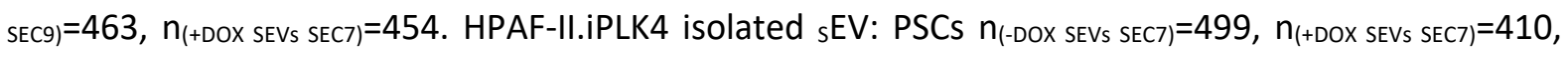
error bars represent mean +/- SD from three independent experiments. ${ }^{* *} p<0.001,{ }^{* * *} p<0.0001$, n.s. $=$ not significant $(p>0.05)$. For all graphs were analyzed using by two-way ANOVA with Tukey's post hoc test. See also Figure S5.

Figure 6. $s E V s$ secreted by cells with extra centrosomes can promote PDAC invasion. (A) Experimental flowchart. (B) Representative brightfield images of heterotypic spheroids. Black arrows: invasive protrusions. Scale bar, $100 \mu \mathrm{m}$. (C) Quantification of the percentage of invasion in 3D spheroids. 5 $\mathrm{ng} / \mathrm{ml} \mathrm{TGF-} \beta$ was used as positive control. Spheroids $\mathrm{n}_{(+\mathrm{PSCS})}=40, \mathrm{n}_{(+\mathrm{PSCS} \text { TGF- } \beta)}=34, \mathrm{n}_{(+\mathrm{PSCS}-\mathrm{DOX} \text { SEVs })}=31$ and $n_{(+P S C s}+D O X$ SEVs) $=31$. (D) Confocal images of spheroids composed of cancer cells (expressing $\mathrm{H}_{2} B-R F P$; magenta) and PSCs (expressing $\mathrm{H}_{2} \mathrm{~B}-\mathrm{GFP}$; green). Scale bar, $100 \mu \mathrm{m}$. Insect depicts higher 
magnification of invasive protrusion. Scale bar, $20 \mu \mathrm{m}$. For all graphics error bars represent mean +/-

736 SD from three independent experiments. ${ }^{* * * *} p<0.0001$, n.s. $=$ not significant $(p>0.05)$. Graph was analyzed using one-way ANOVA with a Kruskal-Wallis post hoc test.

Figure 7. Schematic representation of working model. Increased ROS levels in cells with extra centrosomes compromises lysosomal function. We propose that this changes MVBs fate towards fusing with the plasma membrane and secretion of ${ }_{s} E V s$. $s E V s$ secreted by cancer cells with extra centrosomes are functionally distinct and can induce PSCs activation to promote cell invasion. 


\section{References}

1. Chan, J.Y. (2011). A clinical overview of centrosome amplification in human cancers. Int J Biol Sci 7, 1122-1144.

764 2. Zyss, D., and Gergely, F. (2009). Centrosome function in cancer: guilty or innocent? Trends Cell Biol 19, 334-346.

3. Basto, R., Brunk, K., Vinadogrova, T., Peel, N., Franz, A., Khodjakov, A., and Raff, J.W. (2008). Centrosome amplification can initiate tumorigenesis in flies. Cell 133, 1032-1042.

4. $\quad$ Levine, M.S., Bakker, B., Boeckx, B., Moyett, J., Lu, J., Vitre, B., Spierings, D.C., Lansdorp, P.M., Cleveland, D.W., Lambrechts, D., et al. (2017). Centrosome Amplification Is Sufficient to Promote Spontaneous Tumorigenesis in Mammals. Developmental cell 40, 313-322 e315.

5. Coelho, P.A., Bury, L., Shahbazi, M.N., Liakath-Ali, K., Tate, P.H., Wormald, S., Hindley, C.J., Huch, M., Archer, J., Skarnes, W.C., et al. (2015). Over-expression of Plk4 induces centrosome amplification, loss of primary cilia and associated tissue hyperplasia in the mouse. Open Biol 5, 150209.

6. Sercin, O., Larsimont, J.C., Karambelas, A.E., Marthiens, V., Moers, V., Boeckx, B., Le Mercier, M., Lambrechts, D., Basto, R., and Blanpain, C. (2016). Transient PLK4 overexpression accelerates tumorigenesis in p53-deficient epidermis. Nat Cell Biol 18, 100-110.

7. Ganem, N.J., Godinho, S.A., and Pellman, D. (2009). A mechanism linking extra centrosomes to chromosomal instability. Nature 460, 278-282.

780 8. Godinho, S.A., and Pellman, D. (2014). Causes and consequences of centrosome abnormalities in cancer. Philosophical transactions of the Royal Society of London. Series B, Biological sciences 369.

9. Silkworth, W.T., Nardi, I.K., Scholl, L.M., and Cimini, D. (2009). Multipolar spindle pole coalescence is a major source of kinetochore mis-attachment and chromosome missegregation in cancer cells. PLoS One 4, e6564.

10. Arnandis, T., Monteiro, P., Adams, S.D., Bridgeman, V.L., Rajeeve, V., Gadaleta, E., Marzec, J., Chelala, C., Malanchi, I., Cutillas, P.R., et al. (2018). Oxidative Stress in Cells with Extra Centrosomes Drives Non-Cell-Autonomous Invasion. Developmental cell 47, 409-424 e409.

11. Place, A.E., Jin Huh, S., and Polyak, K. (2011). The microenvironment in breast cancer progression: biology and implications for treatment. Breast Cancer Res 13, 227.

12. Quail, D.F., and Joyce, J.A. (2013). Microenvironmental regulation of tumor progression and metastasis. Nat Med 19, 1423-1437.

13. van Niel, G., D'Angelo, G., and Raposo, G. (2018). Shedding light on the cell biology of extracellular vesicles. Nat Rev Mol Cell Biol 19, 213-228.

14. Costa-Silva, B., Aiello, N.M., Ocean, A.J., Singh, S., Zhang, H., Thakur, B.K., Becker, A., Hoshino,

A., Mark, M.T., Molina, H., et al. (2015). Pancreatic cancer exosomes initiate pre-metastatic niche formation in the liver. Nat Cell Biol 17, 816-826.

15. Hurwitz, S.N., Rider, M.A., Bundy, J.L., Liu, X., Singh, R.K., and Meckes, D.G., Jr. (2016). Proteomic profiling of $\mathrm{NCl}-60$ extracellular vesicles uncovers common protein cargo and cancer type-specific biomarkers. Oncotarget 7, 86999-87015.

16. Xu, R., Rai, A., Chen, M., Suwakulsiri, W., Greening, D.W., and Simpson, R.J. (2018). Extracellular vesicles in cancer - implications for future improvements in cancer care. Nat Rev Clin Oncol 15, 617-638.

804 17. Webber, J., Steadman, R., Mason, M.D., Tabi, Z., and Clayton, A. (2010). Cancer exosomes trigger fibroblast to myofibroblast differentiation. Cancer Res 70, 9621-9630.

18. Webber, J.P., Spary, L.K., Sanders, A.J., Chowdhury, R., Jiang, W.G., Steadman, R., Wymant, J., Jones, A.T., Kynaston, H., Mason, M.D., et al. (2015). Differentiation of tumour-promoting stromal myofibroblasts by cancer exosomes. Oncogene 34, 290-302. 
19. Ringuette Goulet, C., Bernard, G., Tremblay, S., Chabaud, S., Bolduc, S., and Pouliot, F. (2018). Exosomes Induce Fibroblast Differentiation into Cancer-Associated Fibroblasts through TGFbeta Signaling. Mol Cancer Res 16, 1196-1204.

812 20. Mohammadi, H., and Sahai, E. (2018). Mechanisms and impact of altered tumour mechanics. Nat Cell Biol 20, 766-774.

21. Fujita, H., Ohuchida, K., Mizumoto, K., Nakata, K., Yu, J., Kayashima, T., Cui, L., Manabe, T., Ohtsuka, T., and Tanaka, M. (2010). alpha-Smooth Muscle Actin Expressing Stroma Promotes an Aggressive Tumor Biology in Pancreatic Ductal Adenocarcinoma. Pancreas 39, 1254-1262.

22. McCarroll, J.A., Naim, S., Sharbeen, G., Russia, N., Lee, J., Kavallaris, M., Goldstein, D., and Phillips, P.A. (2014). Role of pancreatic stellate cells in chemoresistance in pancreatic cancer. Frontiers in physiology 5, 141.

23. Kadaba, R., Birke, H., Wang, J., Hooper, S., Andl, C.D., Di Maggio, F., Soylu, E., Ghallab, M., Bor, D., Froeling, F.E., et al. (2013). Imbalance of desmoplastic stromal cell numbers drives aggressive cancer processes. J Pathol 230, 107-117.

24. Zhang, Y.F., Zhou, Y.Z., Zhang, B., Huang, S.F., Li, P.P., He, X.M., Cao, G.D., Kang, M.X., Dong, X., and Wu, Y.L. (2019). Pancreatic cancer-derived exosomes promoted pancreatic stellate cells recruitment by pancreatic cancer. J Cancer 10, 4397-4407.

25. Headland, S.E., Jones, H.R., D'Sa, A.S., Perretti, M., and Norling, L.V. (2014). Cutting-edge analysis of extracellular microparticles using ImageStream(X) imaging flow cytometry. Sci Rep 4, 5237.

26. Godinho, S.A., Picone, R., Burute, M., Dagher, R., Su, Y., Leung, C.T., Polyak, K., Brugge, J.S., Thery, M., and Pellman, D. (2014). Oncogene-like induction of cellular invasion from centrosome amplification. Nature 510, 167-171.

27. Kowal, J., Tkach, M., and Thery, C. (2014). Biogenesis and secretion of exosomes. Curr Opin Cell Biol 29, 116-125.

28. Colombo, M., Moita, C., van Niel, G., Kowal, J., Vigneron, J., Benaroch, P., Manel, N., Moita, L.F., Thery, C., and Raposo, G. (2013). Analysis of ESCRT functions in exosome biogenesis, composition and secretion highlights the heterogeneity of extracellular vesicles. J Cell Sci 126, 5553-5565.

29. Mardakheh, F.K., Sailem, H.Z., Kumper, S., Tape, C.J., McCully, R.R., Paul, A., AnjomaniVirmouni, S., Jorgensen, C., Poulogiannis, G., Marshall, C.J., et al. (2016). Proteomics profiling of interactome dynamics by colocalisation analysis (COLA). Mol Biosyst 13, 92-105.

30. Boing, A.N., van der Pol, E., Grootemaat, A.E., Coumans, F.A., Sturk, A., and Nieuwland, R. (2014). Single-step isolation of extracellular vesicles by size-exclusion chromatography. J Extracell Vesicles 3.

31. Muller, L., Hong, C.S., Stolz, D.B., Watkins, S.C., and Whiteside, T.L. (2014). Isolation of biologically-active exosomes from human plasma. J Immunol Methods 411, 55-65.

32. Kalra, H., Simpson, R.J., Ji, H., Aikawa, E., Altevogt, P., Askenase, P., Bond, V.C., Borras, F.E., Breakefield, X., Budnik, V., et al. (2012). Vesiclepedia: a compendium for extracellular vesicles with continuous community annotation. PLoS Biol 10, e1001450.

33. Harding, C., Heuser, J., and Stahl, P. (1983). Receptor-mediated endocytosis of transferrin and recycling of the transferrin receptor in rat reticulocytes. The Journal of cell biology 97, 329339.

852 34. Alvarez-Erviti, L., Seow, Y., Schapira, A.H., Gardiner, C., Sargent, I.L., Wood, M.J., and Cooper, J.M. (2011). Lysosomal dysfunction increases exosome-mediated alpha-synuclein release and transmission. Neurobiol Dis 42, 360-367.

35. Latifkar, A., Ling, L., Hingorani, A., Johansen, E., Clement, A., Zhang, X., Hartman, J., Fischbach, C., Lin, H., Cerione, R.A., et al. (2019). Loss of Sirtuin 1 Alters the Secretome of Breast Cancer Cells by Impairing Lysosomal Integrity. Developmental cell 49, 393-408 e397.

36. Miao, Y., Li, G., Zhang, X., Xu, H., and Abraham, S.N. (2015). A TRP Channel Senses Lysosome Neutralization by Pathogens to Trigger Their Expulsion. Cell 161, 1306-1319. 
860 37. Aits, S., and Jaattela, M. (2013). Lysosomal cell death at a glance. J Cell Sci 126, 1905-1912.

38. Nilsson, E., Ghassemifar, R., and Brunk, U.T. (1997). Lysosomal heterogeneity between and within cells with respect to resistance against oxidative stress. Histochem J 29, 857-865.

39. Leung-Toung, R., Li, W., Tam, T.F., and Karimian, K. (2002). Thiol-dependent enzymes and their

40. Yoshimori, T., Yamamoto, A., Moriyama, Y., Futai, M., and Tashiro, Y. (1991). Bafilomycin A1, a specific inhibitor of vacuolar-type $\mathrm{H}(+)$-ATPase, inhibits acidification and protein degradation in lysosomes of cultured cells. J Biol Chem 266, 17707-17712.

41. Savina, A., Furlan, M., Vidal, M., and Colombo, M.I. (2003). Exosome release is regulated by a calcium-dependent mechanism in K562 cells. J Biol Chem 278, 20083-20090.

42. Kobayashi, T., Stang, E., Fang, K.S., de Moerloose, P., Parton, R.G., and Gruenberg, J. (1998). A lipid associated with the antiphospholipid syndrome regulates endosome structure and function. Nature 392, 193-197.

43. Zhitomirsky, B., Farber, H., and Assaraf, Y.G. (2018). LysoTracker and MitoTracker Red are transport substrates of P-glycoprotein: implications for anticancer drug design evading multidrug resistance. J Cell Mol Med 22, 2131-2141.

44. Masamune, A., Yoshida, N., Hamada, S., Takikawa, T., Nabeshima, T., and Shimosegawa, T. (2018). Exosomes derived from pancreatic cancer cells induce activation and profibrogenic activities in pancreatic stellate cells. Biochem Biophys Res Commun 495, 71-77.

45. Erkan, M., Adler, G., Apte, M.V., Bachem, M.G., Buchholz, M., Detlefsen, S., Esposito, I., Friess, 46. Apte, M.V., Park, S., Phillips, P.A., Santucci, N., Goldstein, D., Kumar, R.K., Ramm, G.A., Buchler, M., Friess, H., McCarroll, J.A., et al. (2004). Desmoplastic reaction in pancreatic cancer: role of pancreatic stellate cells. Pancreas 29, 179-187.

47. Sahai, E., Astsaturov, I., Cukierman, E., DeNardo, D.G., Egeblad, M., Evans, R.M., Fearon, D., Greten, F.R., Hingorani, S.R., Hunter, T., et al. (2020). A framework for advancing our understanding of cancer-associated fibroblasts. Nat Rev Cancer 20, 174-186.

48. Labernadie, A., Kato, T., Brugues, A., Serra-Picamal, X., Derzsi, S., Arwert, E., Weston, A., Gonzalez-Tarrago, V., Elosegui-Artola, A., Albertazzi, L., et al. (2017). A mechanically active heterotypic $\mathrm{E}$-cadherin/ $\mathrm{N}$-cadherin adhesion enables fibroblasts to drive cancer cell invasion. Nat Cell Biol 19, 224-237.

49. Shin, H.R., and Zoncu, R. (2020). The Lysosome at the Intersection of Cellular Growth and Destruction. Developmental cell 54, 226-238.

50. Denu, R.A., Kaur, G., Sass, M.M., Lakkaraju, A., and Burkard, M.E. (2020). Centrosome Amplification in Cancer Disrupts Autophagy and Sensitizes to Autophagy Inhibition. Mol

51. Willms, E., Johansson, H.J., Mager, I., Lee, Y., Blomberg, K.E., Sadik, M., Alaarg, A., Smith, C.I., Lehtio, J., El Andaloussi, S., et al. (2016). Cells release subpopulations of exosomes with distinct molecular and biological properties. Sci Rep 6, 22519.

900 52. Tauro, B.J., Greening, D.W., Mathias, R.A., Mathivanan, S., Ji, H., and Simpson, R.J. (2013). Two distinct populations of exosomes are released from LIM1863 colon carcinoma cell-derived organoids. Mol Cell Proteomics 12, 587-598.

53. McKelvey, K.J., Powell, K.L., Ashton, A.W., Morris, J.M., and McCracken, S.A. (2015). Exosomes: Mechanisms of Uptake. J Circ Biomark 4, 7.

54. Buzas, E.I., Toth, E.A., Sodar, B.W., and Szabo-Taylor, K.E. (2018). Molecular interactions at the surface of extracellular vesicles. Semin Immunopathol 40, 453-464.

55. Willms, E., Cabanas, C., Mager, I., Wood, M.J.A., and Vader, P. (2018). Extracellular Vesicle Heterogeneity: Subpopulations, Isolation Techniques, and Diverse Functions in Cancer Progression. Front Immunol 9, 738. 
56. Laulagnier, K., Javalet, C., Hemming, F.J., Chivet, M., Lachenal, G., Blot, B., Chatellard, C., and Sadoul, R. (2018). Amyloid precursor protein products concentrate in a subset of exosomes specifically endocytosed by neurons. Cell Mol Life Sci 75, 757-773.

57. O'Neill, C.P., Gilligan, K.E., and Dwyer, R.M. (2019). Role of Extracellular Vesicles (EVs) in Cell Stress Response and Resistance to Cancer Therapy. Cancers (Basel) 11.

58. Eldh, M., Ekstrom, K., Valadi, H., Sjostrand, M., Olsson, B., Jernas, M., and Lotvall, J. (2010). Exosomes communicate protective messages during oxidative stress; possible role of exosomal shuttle RNA. PLoS One 5, e15353.

59. Li, N.F., Kocher, H.M., Salako, M.A., Obermueller, E., Sandle, J., and Balkwill, F. (2009). A novel function of colony-stimulating factor 1 receptor in hTERT immortalization of human epithelial cells. Oncogene 28, 773-780.

60. Campeau, E., Ruhl, V.E., Rodier, F., Smith, C.L., Rahmberg, B.L., Fuss, J.O., Campisi, J., Yaswen, P., Cooper, P.K., and Kaufman, P.D. (2009). A versatile viral system for expression and depletion of proteins in mammalian cells. PLoS One 4, e6529.

924 61. Schneider, C.A., Rasband, W.S., and Eliceiri, K.W. (2012). NIH Image to ImageJ: 25 years of image analysis. Nat Methods 9, 671-675.

62. Lo Cicero, A., Delevoye, C., Gilles-Marsens, F., Loew, D., Dingli, F., Guere, C., Andre, N., Vie, K., van Niel, G., and Raposo, G. (2015). Exosomes released by keratinocytes modulate melanocyte pigmentation. Nat Commun 6, 7506.

63. Mardakheh, F.K., Paul, A., Kumper, S., Sadok, A., Paterson, H., McCarthy, A., Yuan, Y., and Marshall, C.J. (2015). Global Analysis of mRNA, Translation, and Protein Localization: Local Translation Is a Key Regulator of Cell Protrusions. Developmental cell 35, 344-357.

932 64. Rappsilber, J., Mann, M., and Ishihama, Y. (2007). Protocol for micro-purification, enrichment, pre-fractionation and storage of peptides for proteomics using StageTips. Nat Protoc 2, 18961906.

65. Tyanova, S., Temu, T., and Cox, J. (2016). The MaxQuant computational platform for mass

66. Vizcaino, J.A., Deutsch, E.W., Wang, R., Csordas, A., Reisinger, F., Rios, D., Dianes, J.A., Sun, Z., Farrah, T., Bandeira, N., et al. (2014). ProteomeXchange provides globally coordinated proteomics data submission and dissemination. Nat Biotechnol 32, 223-226. 


\section{Figure 1}

A.

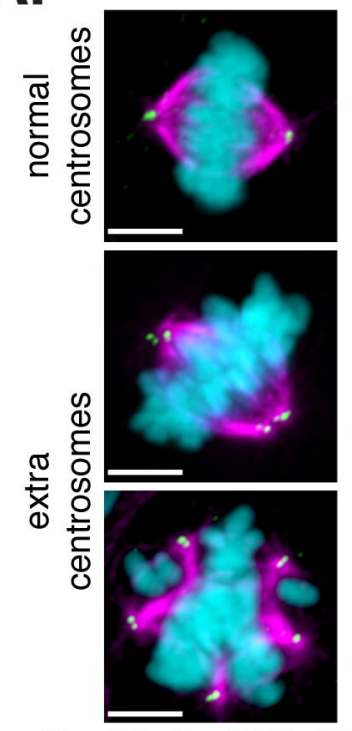

Centrioles/MTs/DNA
B.

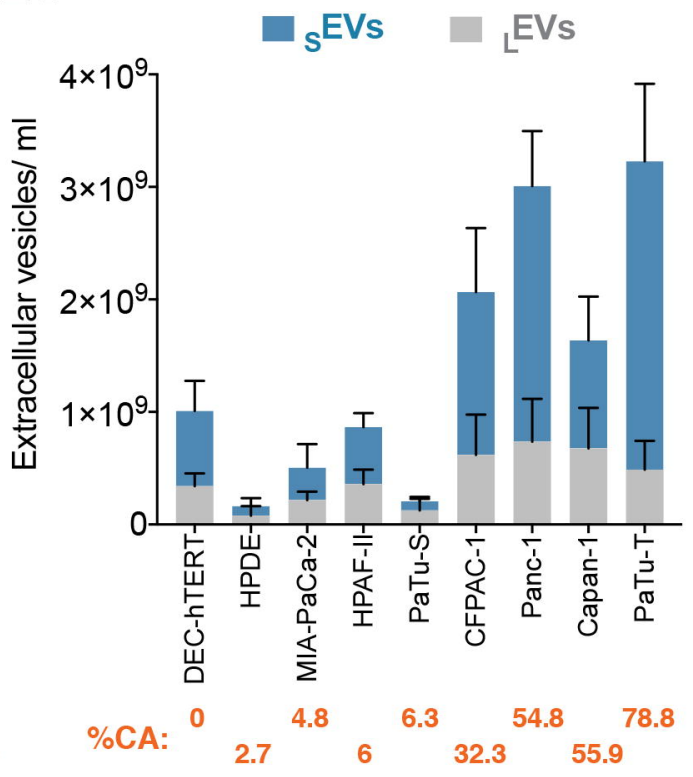

C.

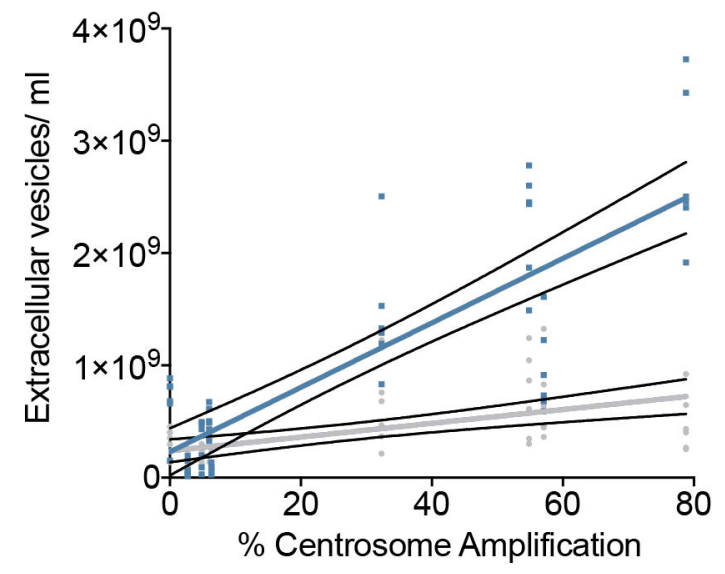

EVs rSpearman: $0.2971 \mathrm{p}:<0.0001$

sEVs rSpearman: 0.6863 p: $<0.0001$

E.

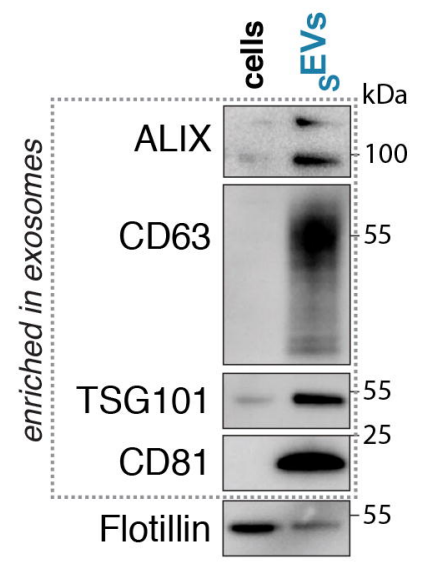

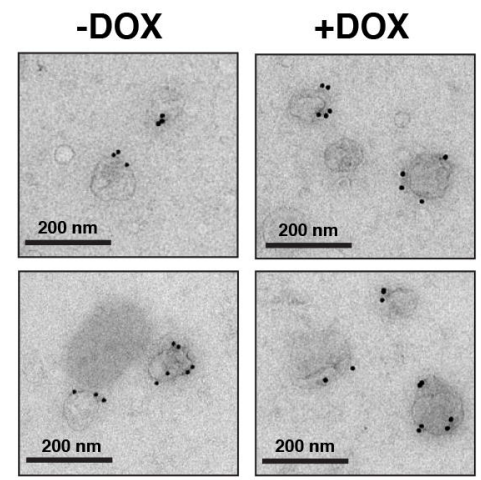

\%CD63 positive sEVs

\begin{tabular}{lll}
\cline { 2 - 3 } & -DOX & + DOX \\
\hline PaTu-S.iPLK4 & $11.63 \%$ & $31.70 \%$ \\
\hline HPAF-II.IPLK4 & $36.48 \%$ & $45.65 \%$ \\
\hline
\end{tabular}

G.

HPAF-II.IPLK4

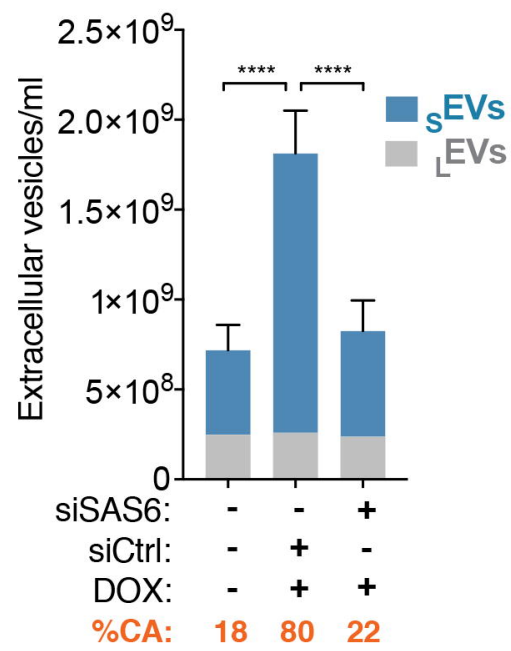

PaTu-S.IPLK4

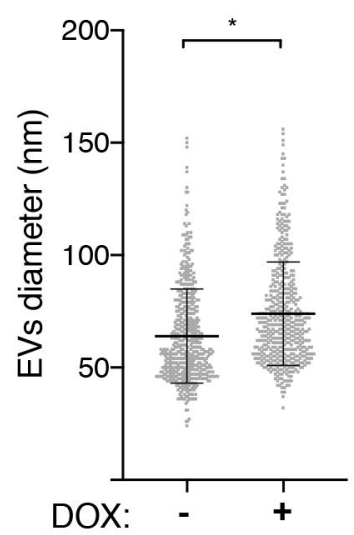

HPAF-II.iPLK4

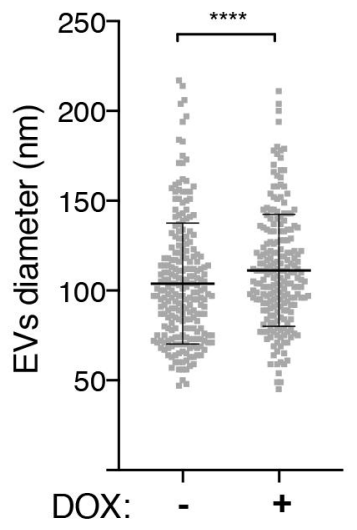


A.
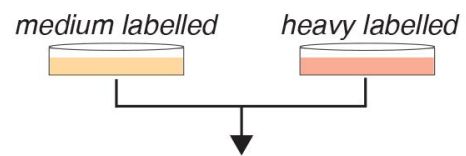

conditioned media

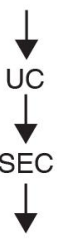

mass spectometry
B.

\section{SEC 8}

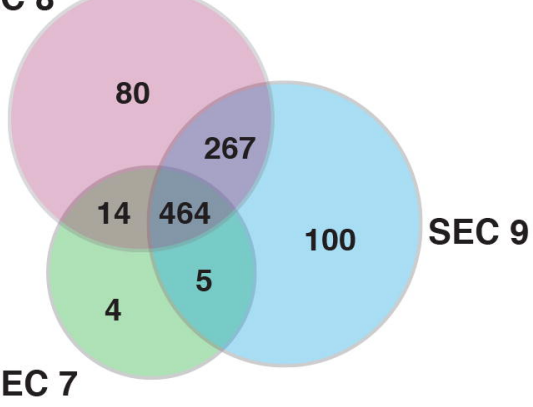

C.

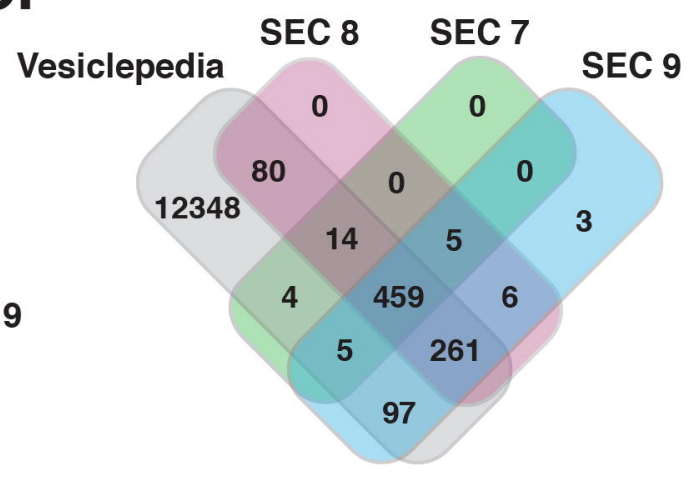

D.

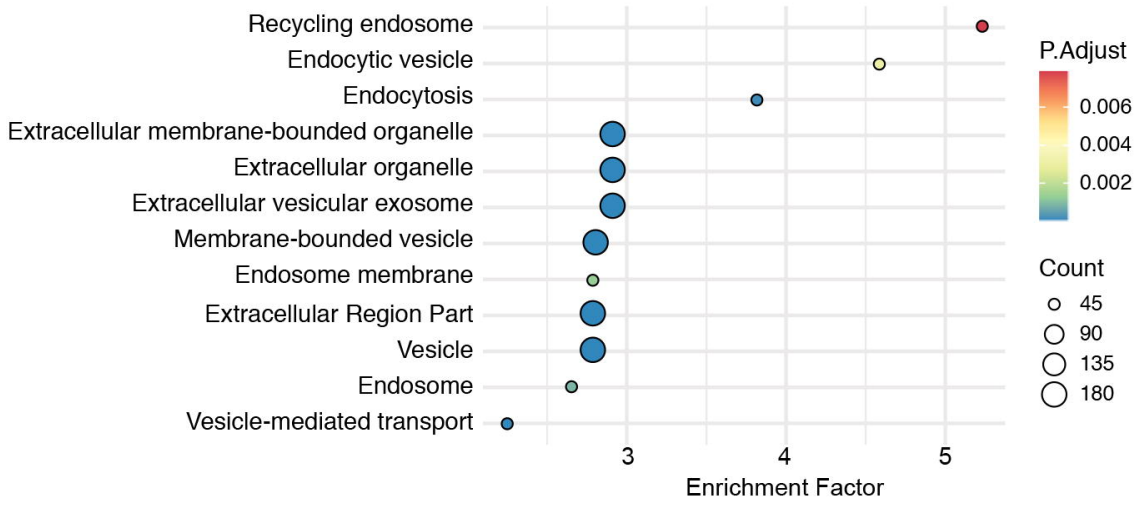

MHC class II protein complex binding MHC protein complex binding

Pancreatic secretion Unfolded protein binding Focal adhesion

Ubiquitin protein ligase binding Cell migration Locomotion Cytoskeletal part Regulation of apoptosis Cytoskeleton

Cellular response to chemical stimulus Immune system process Signal transduction

Regulation of response to stress Regulation of cell communication

Cellular response to stimulus

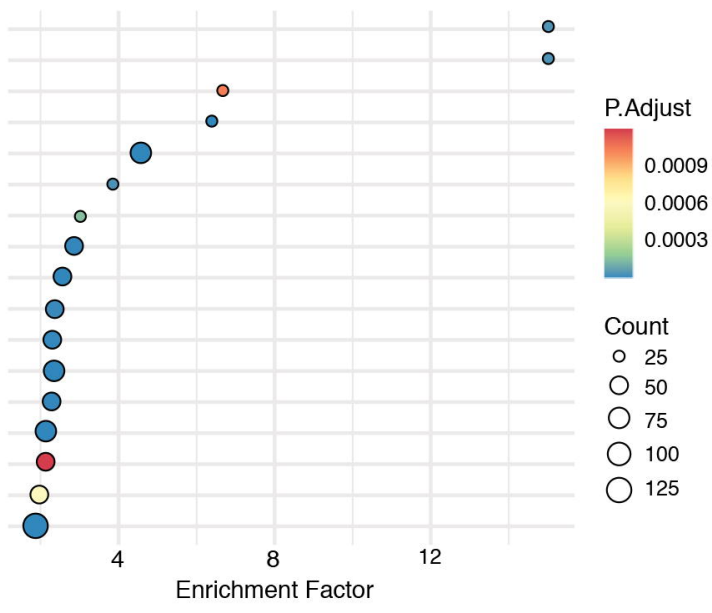


A.

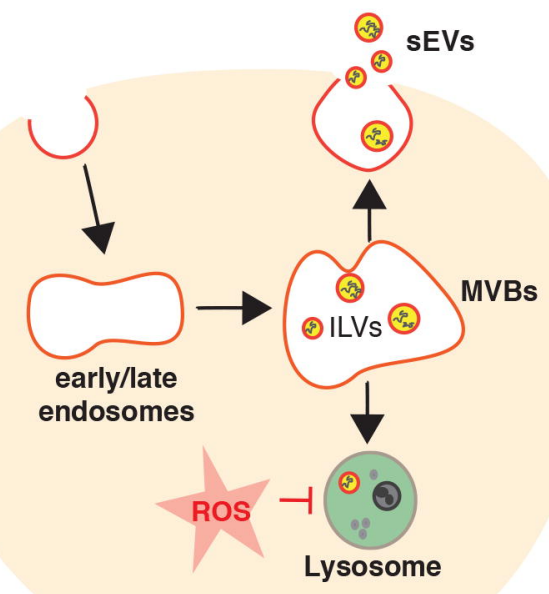

C.
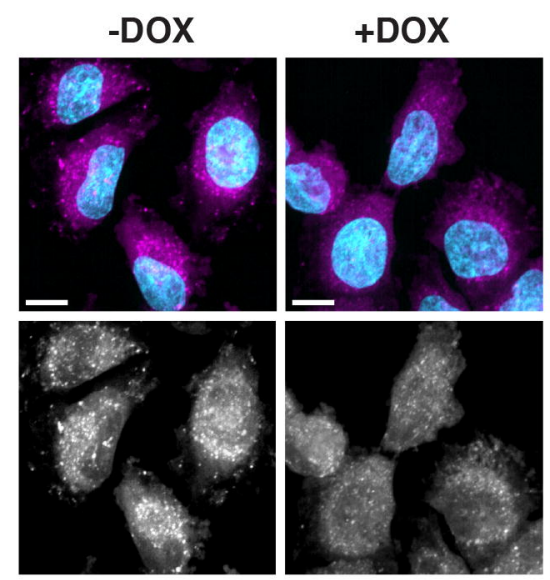

Magic Red/DNA

E.

PaTu-S.iPLK4
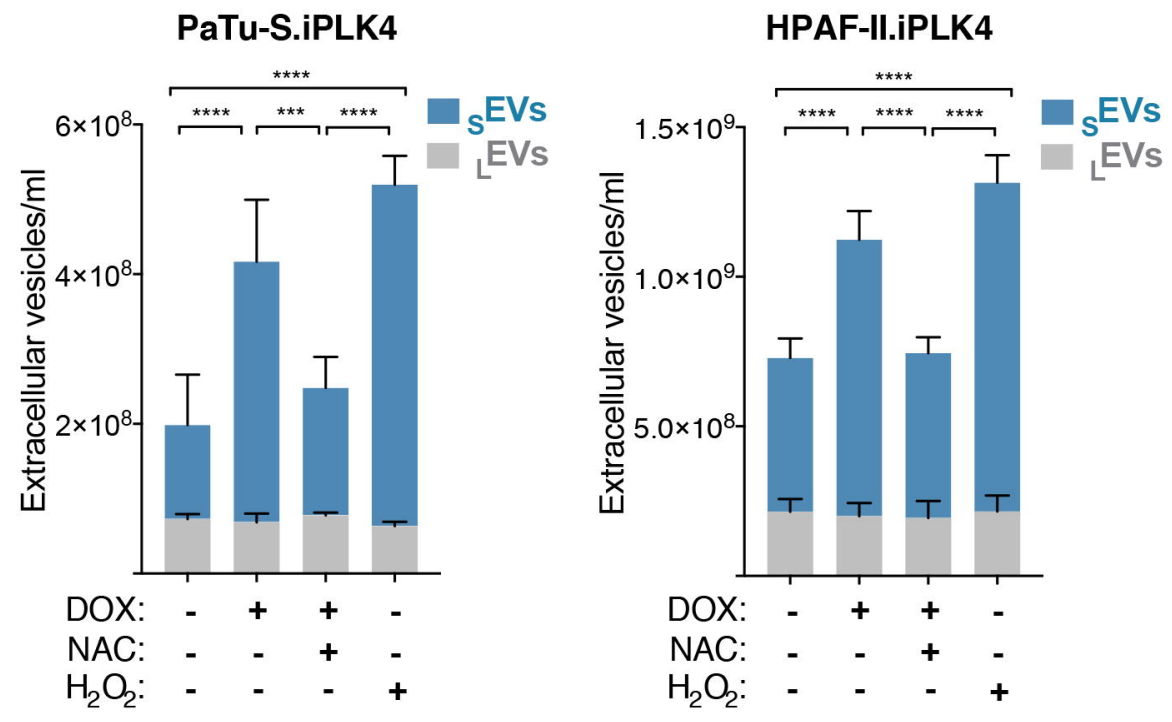

B.
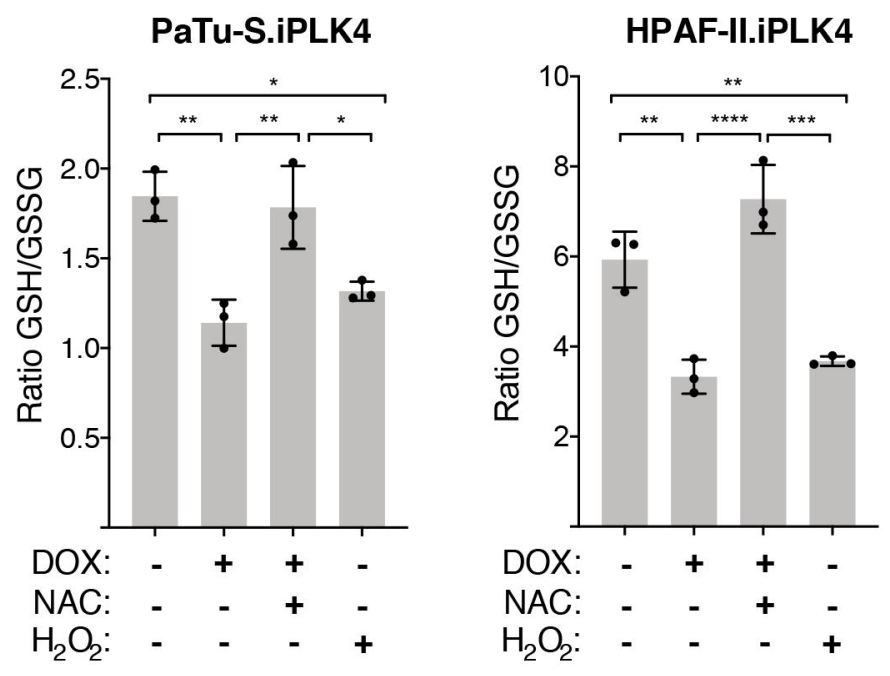

D.

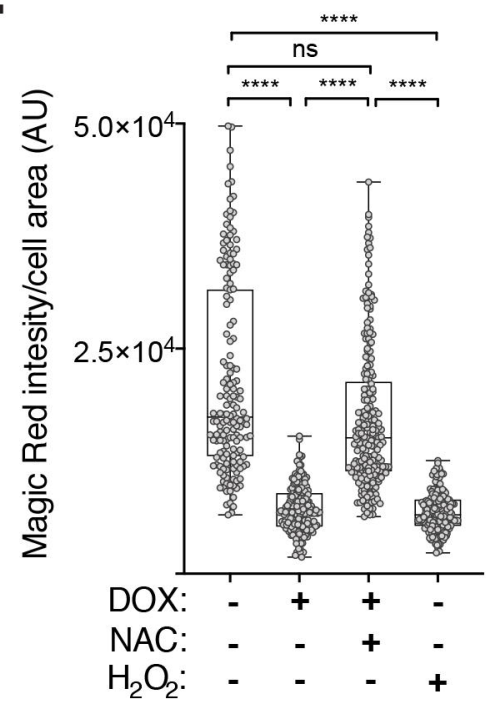

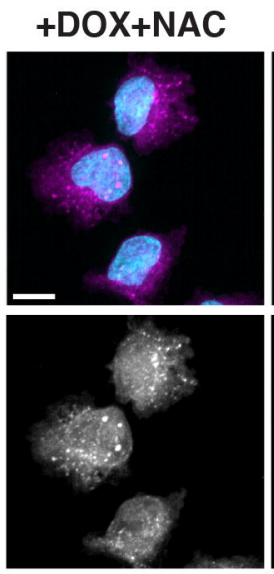

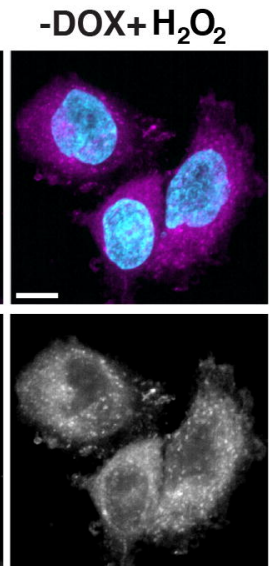

$\mathrm{H}_{2} \mathrm{O}_{2}$ : 
Figure 4

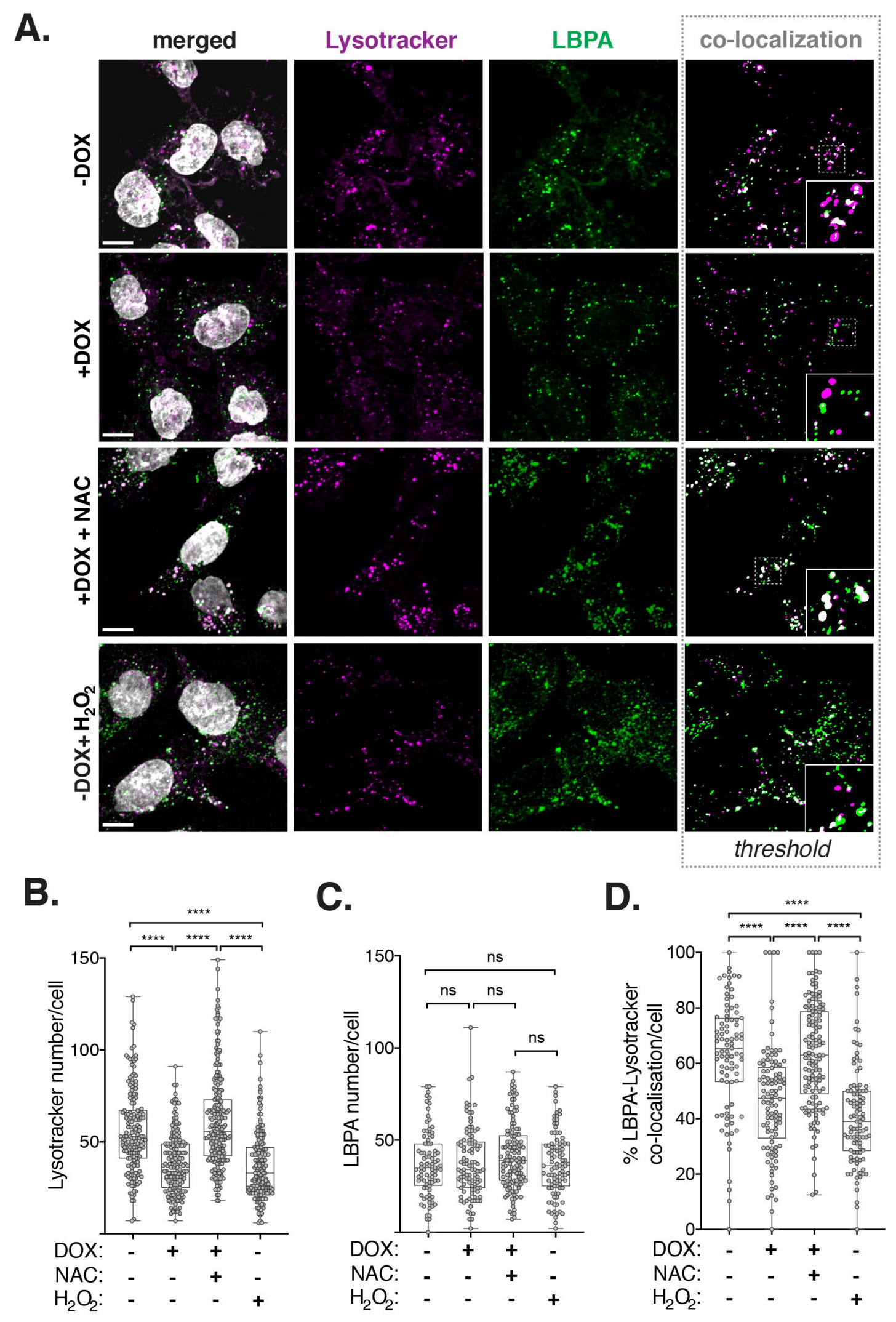




\section{Figure 5}

A.

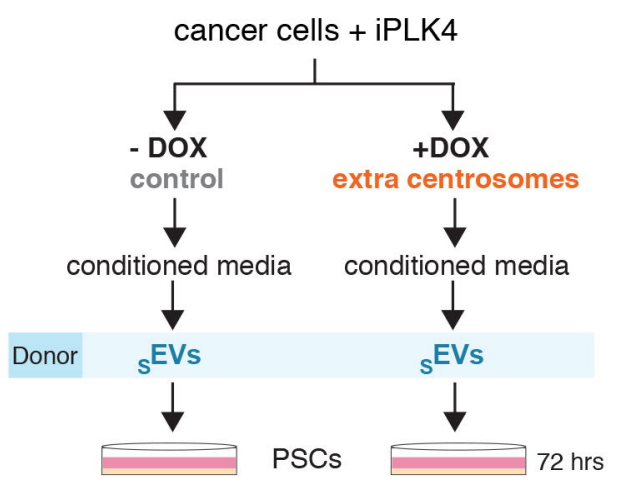

B.

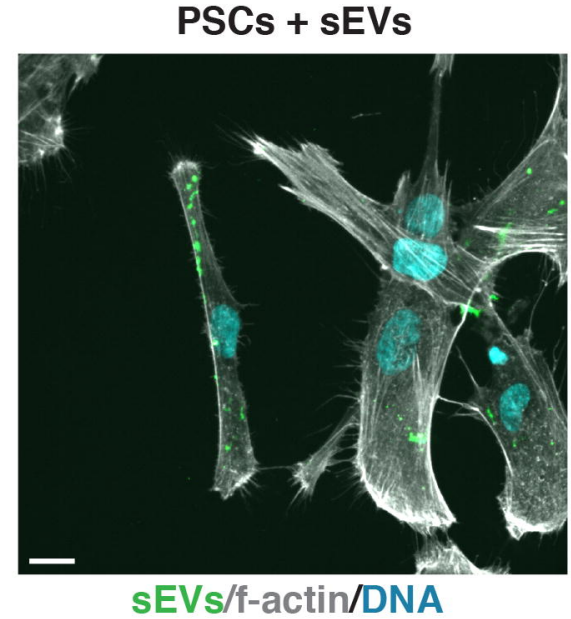

C.
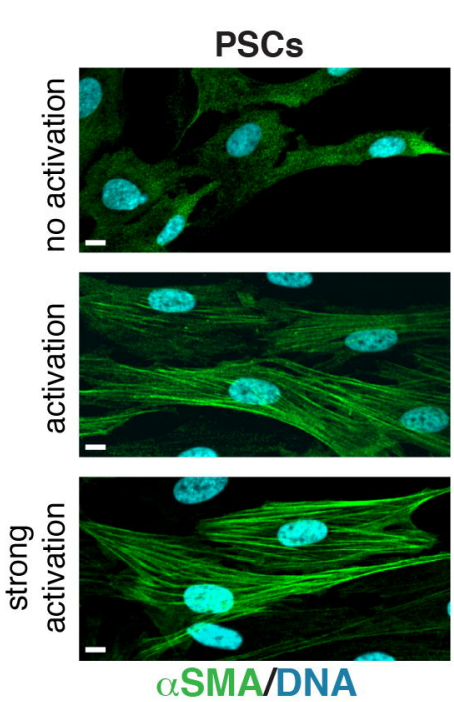

E.

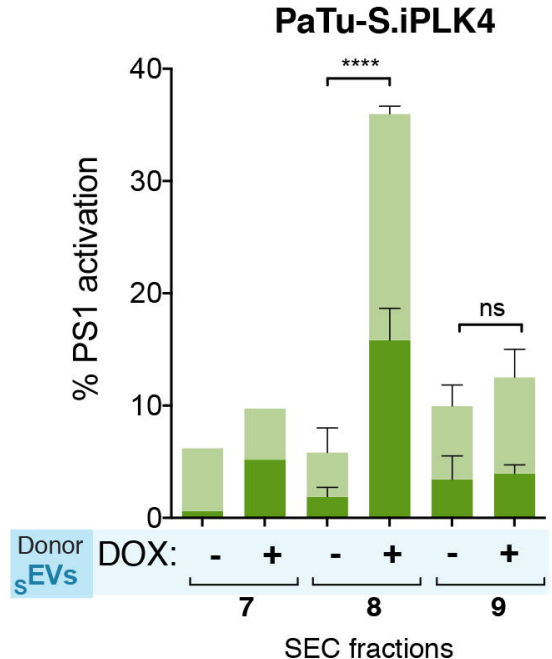

D.

PaTu-S.iPLK4

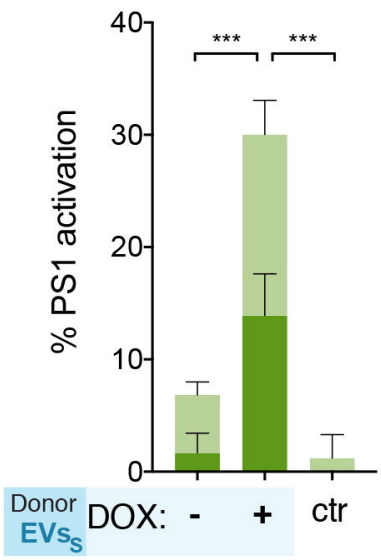

HPAF-II.iPLK4

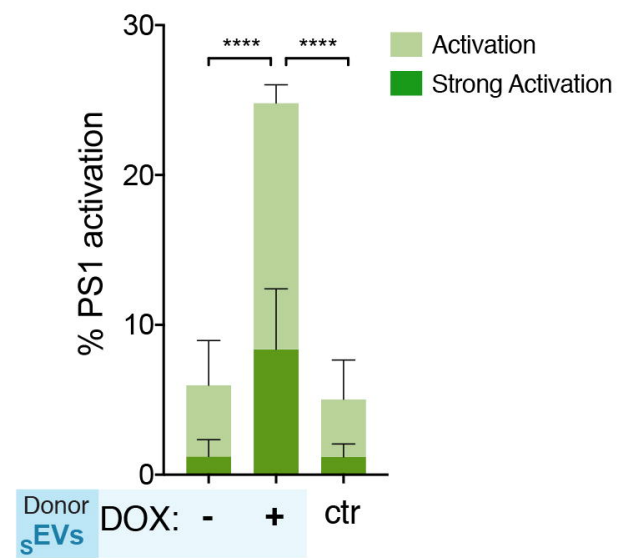

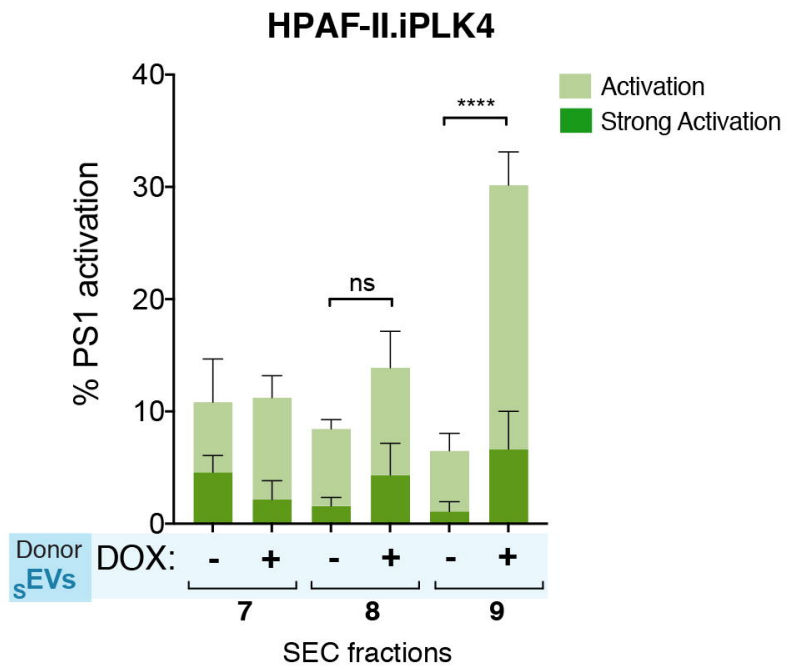




\section{Figure 6}

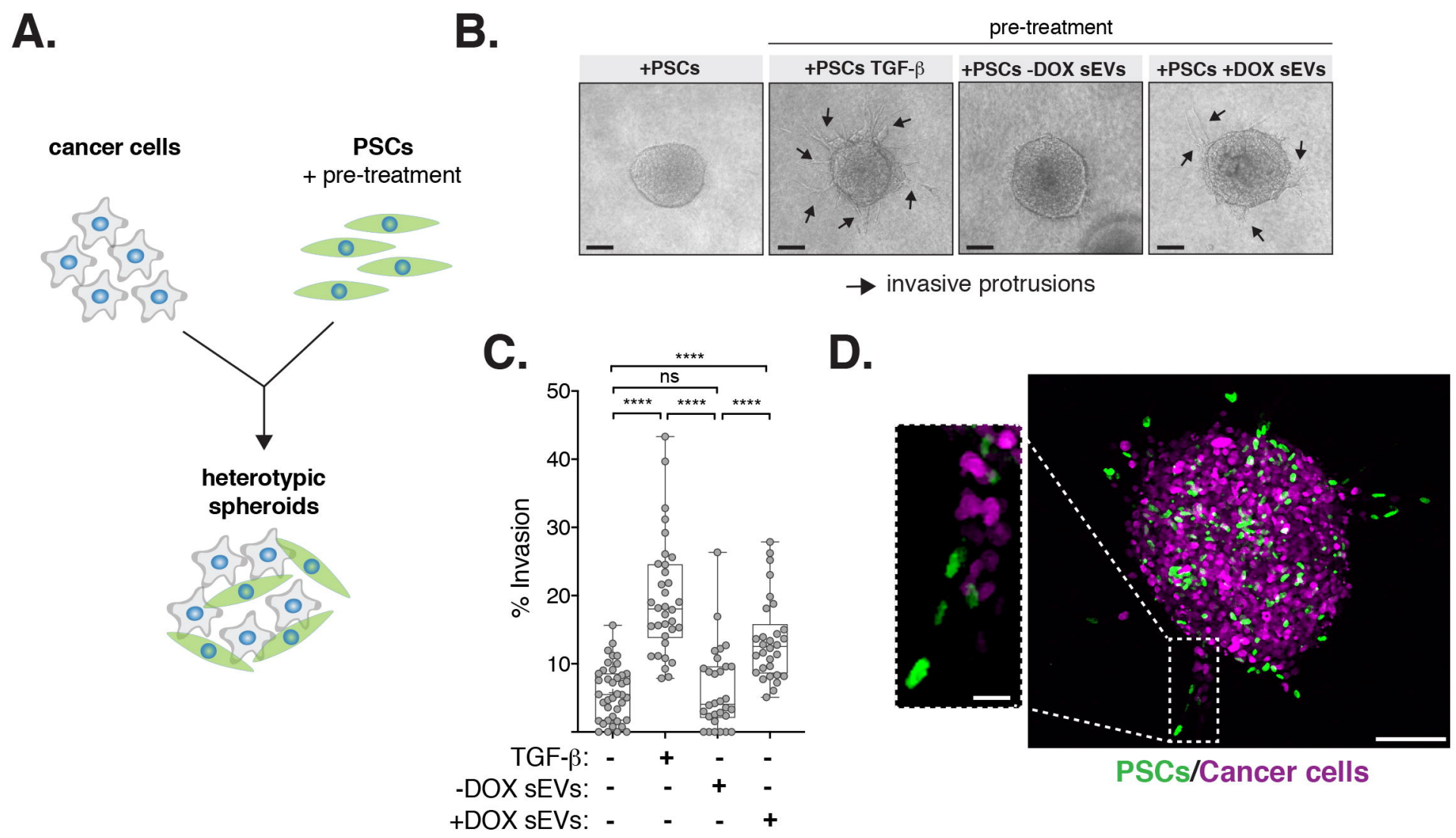


Figure 7

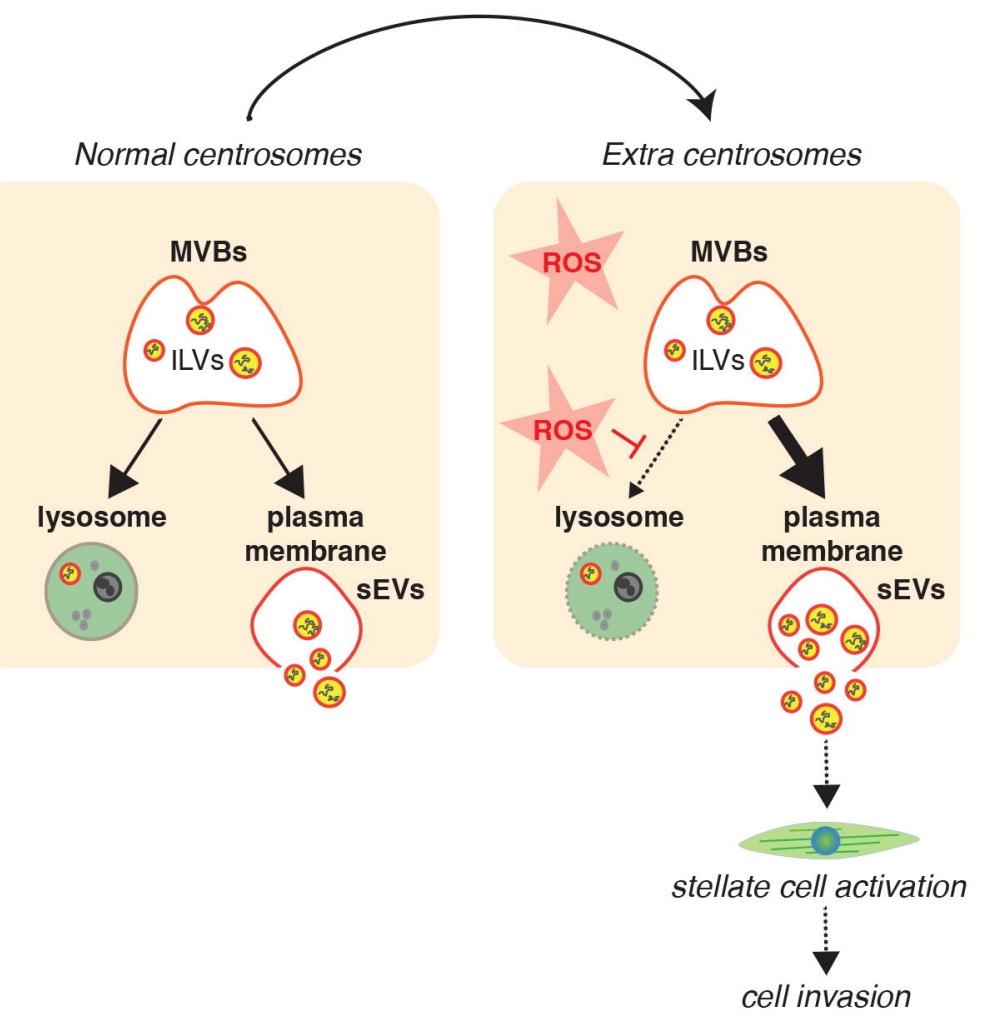

Adams et al 